\title{
On the rate dependent behaviour of epoxy adhesive joints: experimental characterisation and modelling of mode I failure
}

\author{
M. Lißner ${ }^{\mathrm{a}}$, E. Alabort ${ }^{\mathrm{a}}$, H. Cui ${ }^{\mathrm{b}}$, A. Pellegrino ${ }^{\mathrm{a}}$, N. Petrinic ${ }^{\mathrm{a}}$ \\ ${ }^{a}$ Department of Engineering Science, University of Oxford, Parks Road, Oxford, OX1 3PJ, United Kingdom \\ ${ }^{b}$ School of Aerospace, Transport and Manufacturing, Cranfield University, Bedford, MK43 0AL, United Kingdom
}

\begin{abstract}
The increasing use of adhesive joints in dynamic applications require reliable measurements of the rate-dependent stress-displacement behaviour. The direct measurement of the stress-displacement curve is necessary when using cohesive models in discretised solutions of boundary value problems in solid mechanics. This paper aims to investigate the rate-dependent tensile failure of adhesive joints by using a new experimental methodology - it relies upon the combination of the stress wave propagation theory and digital image correlation methods on high speed footage to quantify the tensile stress and the dissipated energy respectively. For this purpose, the Split Hopkinson Bar methodology was employed - the experimental configuration was optimised using numerical modelling. To prove the sensitivity of our framework, two different adhesives are characterised at different loading rates: the adhesive failure strength was found to increase considerably with the strain rate, while the plastic deformation of these adhesives was reduced. The film adhesive showed superior performance over the particle toughened one. In the final part, a rate-dependent cohesive zone model is proposed, one which captures the measured behaviour and which has the potential to be used in industrial applications.
\end{abstract}

Keywords: adhesive joints, rate-dependent, dynamic characterisation, cohesive zone model

\section{Introduction}

Composite materials are increasingly used in modern aerospace [1] and automotive structures 2]. The increased integration level of composite structures is beneficial to its affordability and development efficiency; adhesive bonding has been widely used for manufacturing integrated composite structures [3]. Some typical composite joints have been comprehensively investigated, such as single lap joints [4, double lap joints [5] and T-joints [6]. The strength and damage tolerance 
of those adhesive bonded structures have been extensively documented; the experimental characterization methods [7, 8, 9] have been well established, and corresponding numerical models have been successfully developed [10, 11, 12]. However, the dynamic response of adhesive bonding has not been fully understood yet [2, 13, although many adhesively bonded structures are threatened by impact loading [14].

The analysis of the failure process relies upon the measurement of two mechanical properties: (i) the material strength (critical stress) and (ii) the fracture toughness $\left(G_{C}, K_{C}\right)$. For an accurate failure prediction of adhesive joints, it is essential to find an appropriate way to measure these characteristic parameters with appropriate precision [13]. Traditionally, the fracture behaviour of adhesive joints in mode I loading direction has been characterised in a quasi-static fashion; generally using the double cantilever beam (DCB) [7, 15, 16] and the tapered double cantilever beam (TDCB) [17. However, these methods are very challenging at high loading rates - i.e. 1 to $10 \mathrm{~m} / \mathrm{s}$ - the data captured is usually very noisy due to dynamic effects. Filtering and data reduction methods that may introduce inaccuracies could be necessary. In the case of high-rate loading, the mechanical properties of adhesive joints have been measured using pendulum testing machines [18, 19]; these are based on the Izod and Charpy techniques [20, while Kumar et.al. 21] determined the dynamic fracture toughness using gun impact experiments. In this case the velocity was measured using laser extensometry, thus allowing the estimation of $G_{C}$ using the standard methods for quasistatic loading. Although, they provide a useful comparative ranking these results may not be reliable for the purpose of characterising the dynamic fracture toughness [22]. In order to overcome these limitations, the Split Hopkinson Bar (SHB) apparatus becomes an important tool for direct measurement of stress-displacement relationship across adhesively bonded interfaces.

Even within this well-established experimental framework, there is an urgent need to design appropriate specimens and their gripping methodology in order to apply the desired well-defined rectangular tensile loading pulses upon the relatively thin adhesive bond-lines. In this regard, Yokoyama et.al. [23, 24] investigated the rate dependent performance of metal-to-metal adhesive joints by using new specimen geometries: a solid circular butt joint [23] and a top hat-shaped joint 24] specimen. These studies proved the suitability of the SHB to characterise and measure adhesive properties under impact. Although specimen design is important, measuring the displacements and strains remains challenging. Generally, the deformation of the adhesive joints are determined using the classical SHB analysis $25,26,27,28,29$. . However, it has already been shown that the adhesive 
deformation estimated using the classical SHB analysis might be inaccurate [30, 31. Similar issues occur when determining the dissipated energy - its accuracy relies directly upon the measurement of the local displacements [32, 33. Therefore, there is a need for a direct and reliable measurement of the local strain, for example, employing digital image correlation on high-speed photographic footage. Neumayer et.al. 34 first compared the calculation of the adhesive deformation between the analytical SHB theory and a DIC system - this system highly increased the accuracy of the measurements.

For the engineering and structural design of bonded engineering components, the use of numerical modelling and simulations is critical. These computational tools can be used to predict the behaviour of bonded structures in order to verify their suitability. The commonly adopted approach to model the mechanical behaviour of adhesive joints is the cohesive zone model (CZM) [10, 11, 12. The CZM is a fracture mechanical approach where the progressive damage and failure are represented using cohesive elements [35, 36, 37] which relate the distribution of traction stresses to that of separation displacements across the interface surface. Since the adhesive layer is thin compared to the global structure, the failure process zone of an adhesively bonded joint can be modelled using the cohesive approach. Therefore, the mechanical response of the adhesive must be described by a traction separation law (TSL) [38. Using this relationship, it is possible to couple directly the bulk of the neighbouring substrates and the discontinuity of the adhesively bonded interface in order to simulate crack initiation and crack propagation within the adhesive. Such integrated experimental-modelling approach is adopted here in order to assess the suitability of the proposed experimental procedure and to enable the development of a new cohesive law capable of simulating the observed and quantified rate-dependent behaviour of adhesively bonded interfaces.

With the above in mind, the aim of this paper is to present the results of a newly devised experimental methodology for the characterisation of rate-dependent behaviour of adhesively bonded interfaces. Most importantly, the proposed framework is consistent across all the loading regimes of interest, from quasi-static (QS), via medium-rate (MR) to high-rate (HR). Firstly, a suitable specimen configuration for HR loading regime is designed numerically aiming to ensure: (i) impedance matched specimen gripping, (ii) uniform homogeneous surface traction within the adhesive interface and (iii) direct measurement of the separation across the interface until complete fracture. In order to achieve this, the design is assessed using finite element simulations. Secondly, the developed methodology is used to investigate the rate-dependent nature of two different epoxy based 
metal-to-metal joints. Thirdly, further experimental analyses are carried out to determine the effect of adhesive layer thickness, adhesive composition and loading rate on the joint behaviour in terms of stress-displacement relationship and related dissipated energy prior to complete mode I fracture. Finally, high-resolution fractography is included to validate the proposed specimen design and to elucidate the nature of the studied adhesive interface fracture. In addition, a new rate and thickness-dependent analytical model which corresponds well to the observed and quantified behaviour, suitable for implementation into finite element software is proposed.

\section{Materials and Methods}

\subsection{As-received material and bonding procedure}

This study focuses on two commercially available epoxy adhesives: (i) the 3M Scotch-Weld ${ }^{T M}$ AF 163-2OST, and (ii) the 2216 B/A Gray. Both of these are suitable for joining metallic and plastic adherents. The adhesive AF 163-2OST is an epoxy film supported with a glass fibre matt, where the fibres can be larger than $100 \mu \mathrm{m}$. The second one is a two-component, Kaolin powder-toughened epoxy adhesive. The latter was mixed in a volume ratio of 2:3 - base to hardening accelerator - to achieve the most optimal mechanical properties. Here, the titanium alloy Ti-6Al-4V was used as the adherent material.

In order to obtain an optimal adhesive joint, the surfaces of the titanium end-caps were anodised [17. The adhesive was applied between both titanium end-caps using an alignment fixture which ensured an accurate control of the adhesive thickness using shims with the required thickness - see Fig. 1. Three adhesive thickness were chosen: $0.1,0.3$ and $0.5 \mathrm{~mm}$ for the film adhesive and 0.1, 0.5 and $1.0 \mathrm{~mm}$ for the paste adhesive. The paste adhesive specimens (2216 B/A Gray) were cured for $7 \mathrm{~h}$ at $55^{\circ} \mathrm{C}$. The film adhesive specimens (AF 163-2OST) were cured for $1 \mathrm{~h}$ at $120^{\circ} \mathrm{C}$. In both cases, a pressure of 1.4 bar was applied during curing. The adhesive thickness quality was controlled by measuring the deviation of the received adhesive joints to the nominal adhesive thickness - see Fig. 1 (c). A minimum deviation of less than $5 \%$ has been achieved.

\subsection{Experimental setup}

\subsubsection{The design of the experimental setup}

One-dimensional wave theory is often employed to measure remotely the behaviour of materials when deformed rapidly, for example, when subjected to high-strain loading conditions, by means 
of the Split Hopkinson Bar (SHB) apparatus [25]. Although, developed with impact loads in mind, this approach may also be useful when characterising the response of a material subjected to quasistatic loading, when due to the instabilities in the material response of thus loaded specimens, the experiments become dynamic in nature. Indeed, it is the objective of this paper to present how the measurement methodology based on one-dimensional stress wave propagation theory was adapted for the use in capturing short-lived events which occur regardless of the applied loading rate: e.g. as a result of unstable fracture during experiments in which the load is applied at quasi-static and medium rates.

During the failure of the adhesively bonded interface, the energy stored in the specimen can be released rapidly, thus calling for the use of the stress wave propagation based measurement methodology [39]. The design of all relevant measurement systems for capturing the fracture process should consider this approach. In order to achieve this, the traditional medium and low-strain rate setups must be adapted such that: (i) long instrumented bars are used to quantify the release stress wave and (ii) ultra-high-speed-photography based DIC is used to quantify the separation of the adhesively bonded surfaces irrespective of the rate of loading. This offers two main advantages: first, the softening slope can be captured for every proposed loading regime, and second, because the experimentation and analyses are consistent across the three loading regimes, the comparison and study of thus excited rate-dependent behaviour is direct.

The specimen geometry was designed specifically to minimise negative factors which may induce deviation during the stress propagation step. Among others, the impedance of the loading bars and the specimen end-caps were matched by keeping the same diameter and material throughout the whole characterisation setup. As a result, given that the conducted study was concerned with adhesively bonded titanium surfaces, the instrumented long rods were manufactured from Ti-6Al$4 \mathrm{~V}$ alloy. For data acquisition, strain gauges of the type FLA-2-8 from TML attached on the rods were used to record the strain, as this allowed for data to be acquired at a range of acquisition rates, including very high most commonly at 100 Kilo-samples per second in this study. These strain gauges were also used to trigger the high-speed photographic equipment.

\subsubsection{High-rate loading}

In this paper, the results of dynamic experiments were determined using the Split Hopkinson Tensile Bar (SHTB) apparatus presented in [40]. In the experimental setup, a hollow striker bar 
rides along the input bar and hits an anvil on the end of the input bar to produce the tensile load as illustrated in Fig. 2(a). On the other side of the specimen, the output bar is placed to record the strain-time history as acquired by the strain gauges, in a Wheatstone bridge connected to an oscilloscope.

The input and output bars of length $3 \mathrm{~m}$ and diameter $10 \mathrm{~mm}$ were manufactured from a Ti$6 \mathrm{Al}-4 \mathrm{~V}$ alloy with the density of $\varrho=4.43 \times 10_{-6} \mathrm{~kg} / \mathrm{mm}^{3}$ and the Young's modulus of $\mathrm{E}=114$ GPa. The striker, also made of Ti-6Al-4V and with a length of $2.7 \mathrm{~m}$, was accelerated so that the the relative velocity at the two opposite ends of the specimen was approximately $v \approx 3000 \mathrm{~mm} / \mathrm{s}$. Both input and output bars were instrumented with strain gauges. The wave signals were amplified and recorded using an oscilloscope. For the data analysis, only the strain gauges positioned at the output bar are required. Additionally, the deformation of the adhesive was recorded using a Kirana high-speed camera. The camera was set up to record 500,000 fps and 100,000 fps frames per second, for the film adhesive AF 163-2OST and the paste adhesive 2266 B/A Gray respectively, taking 924 x 768 pixels resolution images with $2.0 \mu$ s or $10.0 \mu$ s interframe between each one. The surfaces along the circumference of the end caps were speckled to evaluate the separation of the adhesively bonded surface by digital image correlation (DIC) using 4M GOM Aramis - see Fig. 11(c). Since the stiffness of the adherents is 15 times higher than the stiffness of the adhesive, the deformation of the adherents can be assumed to be minimal. Moreover, the measuring points for the DIC are close to the adhesive interface. Therefore, it is believed that the measured displacement represents the true deformation of the interface.

\subsubsection{Medium-rate loading}

The medium-rate tensile tests were performed using an Instron servo-hydraulic machine as shown in Fig. 2(b). The applied velocity on the specimen was $v=10 \mathrm{~mm} / \mathrm{s}$. A long bar was employed to be able to capture the falling edge of the strain history. Analogously to the HR tests, a camera setup was used for strain measurement. For measuring displacements across the bonded interface, during the loading step, a SA 5 Photron high-speed camera (at 65,100 fps) was used to record 512 x 408 pixels resolution images. To measure the displacement during the fracture event (softening slope), a Kirana high-speed camera at 1,000,000 fps was employed which recorded images with 924 x 768 pixels resolution. The results of data and image acquisition are illustrated in Fig. 2(c). 


\subsubsection{Quasi-static loading}

The quasi-static tensile tests were performed using a Zwick screw-driven machine. The specimens were loaded using a speed of $v=0.05 \mathrm{~mm} / \mathrm{s}$. A similar setup to the MR tests was used with a long rod strain gauged with an arbitrary distance away from the specimen and two cameras (Fig. 2(b)). For measuring displacement during the loading step, a prism-based line scan Jai camera (5 fps) to record $608 \times 840$ pixel resolution images was used. To capture the displacement during the fracture event (softening slope), a Kirana high-speed camera at 200,000 fps was employed.

\subsection{Data analysis: deriving the stress-displacement response}

The procedure to derive the adhesive joint response is similar for all three loading rates. The main difference is the number of cameras which are used to capture different parts of the stressdisplacement curve. For MR and QS tests, two cameras must be employed, one at a slow frame rate to capture the loading phase and a second one at an ultra-fast frame rate to capture the failure. For HR, a single camera is used to capture the whole event. The design of the experimental setup enables all three loading regimes to rely upon the same measurement methodology for capturing the fracture softening behaviour.

Since different cameras are used to capture different parts of a single test, a careful synchronisation procedure is necessary. For the loading part in MR and QS tests, the synchronisation is trivial - both the mechanical test and the imaging acquisition start at the same time. The displacement is measured using the extensometer tool in Aramis using two points close to the adhesive interface as it is illustrated in Fig]1(c). For the whole HR testing and failure events in QS and MR tests, one must know the exact instant when the tested sample fails in order to trigger the high-speed cameras. For this purpose, the falling slope of the load which occurs upon sample failure is used. The relevant steps for generating the stress-displacement curve are demonstrated in Fig. 3 . A typical strain gauge signal is presented in Fig. 3 (a). However, because the strain gauge which triggers the cameras is positioned at an arbitrary distance from the sample, the signal shown in Fig. 3(a) must be delayed by a time $t_{\text {delay }}$. This is the time required by the stress wave - which starts from the sample itself - to reach the strain gauge. This can be compensated using the expression

$$
t_{\text {delay }}=\frac{l}{c}
$$

where $l$ is the distance of the strain gauge and $c$ is the stress wave propagation speed of the bar 
which can be written in terms of density $\rho$ and elastic modules $E$ following

$$
c=\sqrt{\frac{E}{\rho}} .
$$

The force is estimated with the signal from the output bar using

$$
F=\frac{S G}{A F \cdot C F}
$$

were the signal $S G$ is that captured by the output bar strain gauge in Volts, $A F$ is its amplification factor, 200 for strain gauges 1 and 2 (on the input bar), and 500 for strain gauge 3 on the output bar. $C F=7.5 \times 10^{-7} \mathrm{~V} / \mathrm{N}$ is the calibration factor of the employed strain gauge station. The amplification factor is higher for the transmitted wave (SG3) as the transmitted force is typically lower in magnitude than the applied incident wave (SG1 and 2). The noticeable length of the projectile (equal to the length of the input bar) inevitably leads to the superimposition of incident and reflected elastic stress waves into the input bar at the gauge location. A post-processing procedure, based on the method of characteristics and on D' Alambert's solution of wave equations, was used to calculate the magnitude of forward and backward travelling waves as functions of position and time. Detail on the procedure can be found in [41. The nominal stress applied to the specimen can be derived using the following

$$
\sigma(t)=\frac{F(t)}{A_{0}}
$$

where $\mathrm{F}(\mathrm{t})$ is the force obtained from the strain gauge on the output bar and $A_{0}$ is the initial cross section area of the specimen. Fig 3 (b) shows that force equilibrium is achieved. The displacement separation between the adhesively bonded surfaces was calculated with Aramis using DIC. Fig[3(c) shows the obtained data curve for the displacement and the synchronised force over time. The results of stress and displacement can then be combined in a traditional tensile stress-tensile deformation diagram - see Fig. 3(d).

\subsection{Numerical verification of the experimental setup}

When applying the stress wave propagation theory for the analysis of data generated using the SHB apparatus one needs to be confident that the obtainable results are meaningful. Here, virtual 
experiments are carried out to examine: (i) that the data analyses used during real experimentation are accurate, and (ii) that the specimen geometry is suitable for the proposed investigation.

The accuracy of the measured adhesive response is highly dependent on the homogeneity of tractions along the adhesive interface. More importantly, this homogeneity needs to be maintained throughout the test. To prove the suitability of the available measuring techniques, a numerical verification of the characterisation apparatus was carried out: the experimental setup was simulated using finite element analysis. In our model, the adhesive was simulated using the cohesive zone approach while the rest of the setup was simulated using continuum mechanics. The numerical calculations were conducted using the Abaqus/Explicit solver. The traction separation law (TSL), calculated using the strain information on the bar and the data process methods employed in experiments were compared to the implemented cohesive law. Numerically, the bars and the adherents were modelled as solid bodies using three-dimensional hex volume elements. The adhesive layer was represented as a solid body employing cohesive elements such that it could be directly related to the experimentally obtained cohesive response of the adhesive. The whole model of the virtual experimental setup of the SHB, except the adhesive layer, is assumed as linear elastic and rateindependent. The loading velocity is introduced in the z-direction at the beginning of the input bar. Instead of modelling the striker, the impulse was modelled by applying a load with a pre-defined amplitude. The duration of the striker impulse was defined using

$$
t_{\text {striker }}=\frac{2 L}{c}
$$

where $L$ is the length of the striker and $c$ the stress wave propagation speed within the striker.

\section{Results}

\subsection{A verified experimental setup: numerical design, advantages and limitations}

The specimen design is known to play a key role for generating accurate results. Particularly, the design of the specimen needs to allow a homogeneous traction stress along the adhesively bonded interfaces throughout the tests. Hence, specimens were designed using the same material and cross section than the input and the output bars, so that a perfect acoustic impedance matching is obtained. Virtual experiments demonstrate that a homogeneity across the specimen's surface is achieved - see Fig. 4(a). Additionally, the cohesive response was determined using the two different 
measuring approaches - classical SHB theory and the DIC method. As expected, the comparison with the implemented cohesive law shows no deviation between the two methods. However, as it is well known in literature [34 the use of the SHB analysis (SHBA) tends to overestimate the strain. Fig. 4 (b) supports this statement by presenting the comparison between the experimentally obtained stress-displacement curves measured with the SHBA and the DIC method. The stressdisplacement responses have been extracted in the exact same way as it has been carried out in experiments. As a result, a DIC based method is used during the whole experimental campaign.

The use of long bars for experimenting adhesive joints in QS and MR loading conditions is critical. Both long bars and strain gauges are necessary in order to measure the failure curves see Fig. 5. One can see that without the long bars, an abrupt failure is captured, even under the lower-rate regimes. Since an accurate strain measurement cannot be achieved, a reliable prediction of the whole dissipated energy - which is critical for proposing the corresponding cohesive models is unattainable without employing a more accurate system for measuring the relative displacement of adhesively bonded surfaces during the experiment. Analogously to high-rate loading, a DIC based measurement method was used in experiments with QS and MR loading rates.

The area under the stress-displacement curve gives a measurement of the whole energy which was dissipated during the deformation process. This curve also represents the TSL which is used to derive cohesive models. It is not trivial to distinguish between the specific areas under the obtained curve which quantify the elastic energy, the plastic energy - where voids or cavities start to stretch - and the energy dissipated due to crack coalescence until failure. This last part is also known as the fracture toughness. Fig 6 illustrates an approximation of each of the aforementioned energies. In this paper, the whole energy under the curve is considered as the dissipated energy $G_{\max }$ - this quantity will be used for the subsequent sections.

\subsection{Mode I response of the film adhesive AF 163-2OST 3M Scotch-WeldTM}

The results from a series of tension tests for the film adhesive AF 163-2OST under three different loading rates at room temperature are shown in the left-hand side of Fig. 7 Fig. 8 shows the typical mode I fracture process of a film-adhesive as seen using high-speed cameras. The initial elastic response of all specimens is appreciated as strain-rate independent.

The film adhesive shows a trapezoidal shaped curve for each adhesive thickness. The mean peak stress under QS, MR and HR loading conditions respectively is shown independent from the 
Table 1: Mean properties ( \pm Standard deviation) of the AF 163-2OST adhesive joints during mode I testing under quasi-static, medium-rate and high-rate loading.

\begin{tabular}{ccccc}
\hline & & $\begin{array}{c}\text { Peak stress } \\
{[\mathrm{MPa}]}\end{array}$ & $\begin{array}{c}\text { Final failure displacement } \\
{[\mathrm{mm}]}\end{array}$ & $\begin{array}{c}\text { Dissipated energy } \\
{[\mathrm{N} / \mathrm{mm}]}\end{array}$ \\
\hline$t_{a}=0.1 \mathrm{~mm}$ & QS & $41 \pm 0.9$ & $0.08 \pm 0.01$ & $2.0 \pm 0.7$ \\
& MR & $48 \pm 2.7$ & $0.07 \pm 0.01$ & $2.1 \pm 0.6$ \\
& HR & $59 \pm 1.5$ & $0.05 \pm 0.01$ & $1.7 \pm 0.8$ \\
$t_{a}=0.3 \mathrm{~mm}$ & QS & $36 \pm 0.7$ & $0.25 \pm 0.01$ & $5.4 \pm 0.1$ \\
& MR & $43 \pm 3.9$ & $0.14 \pm 0.04$ & $2.5 \pm 0.5$ \\
& HR & $62 \pm 0.3$ & $0.05 \pm 0.01$ & $1.5 \pm 0.3$ \\
$t_{a}=0.5 \mathrm{~mm}$ & $\mathrm{QS}$ & $38 \pm 1.5$ & $0.21 \pm 0.01$ & \\
& MR & $45 \pm 2.7$ & $0.14 \pm 0.02$ & $3.8 \pm 0.6$ \\
& HR & $55 \pm 2.7$ & $0.06 \pm 0.01$ & $2.1 \pm 0.3$ \\
\hline
\end{tabular}

adhesive layer thickness. The mean final displacement at fracture, $\delta_{\mathrm{f}}$ decreases with the applied load. Moreover, the final displacement tends to increase proportional to the adhesive thickness until a certain threshold for each loading regime is reached. The dissipated energy $\left(G_{\max }\right)$ is calculated as the area under the measured stress-displacement curve. It shows a decrease as the thickness of the adhesive increases up to a certain threshold. The experimental obtained results are shown in Table 1. Since the statistical measurements have been calculated from three repeated tests for each loading rate and loading direction, it is advised to carefully consider the values of the standard deviation.

For the high-rate loading regime, a deviation of the velocity with which the specimens are subjected amounts to $3000 \pm 200 \mathrm{~mm} / \mathrm{s}$.

\subsection{Mode I response of the paste adhesive 2216 B/A Gray 3M Scotch-Weld ${ }^{T M}$}

Analogously, the results from a series of tension tests for the two component paste adhesive 2216 B/A Gray under three different loading rates are shown in the right-hand side of Fig. 7.

The two component paste adhesive shows a bilinear-shaped curve for the HR loading regime 
Table 2: Mean properties ( \pm Standard deviation) of the 2216 B/A Gray adhesive joints during mode I testing under quasi-static, medium-rate and high-rate loading.

\begin{tabular}{ccccc}
\hline & & $\begin{array}{c}\text { Peak stress } \\
{[\mathrm{MPa}]}\end{array}$ & $\begin{array}{c}\text { Final failure displacement } \\
{[\mathrm{mm}]}\end{array}$ & $\begin{array}{c}\text { Dissipated energy } \\
{[\mathrm{N} / \mathrm{mm}]}\end{array}$ \\
\hline$t_{a}=0.1 \mathrm{~mm}$ & QS & $19 \pm 0.1$ & $0.08 \pm 0.02$ & $1.0 \pm 0.4$ \\
& MR & $28 \pm 14.6$ & $0.05 \pm 0.03$ & $1.3 \pm 0.8$ \\
& HR & $41 \pm 8.4$ & $0.03 \pm 0.01$ & $0.8 \pm 0.2$ \\
$t_{a}=0.5 \mathrm{~mm}$ & QS & $16 \pm 0.9$ & $0.26 \pm 0.05$ & $2.1 \pm 0.5$ \\
& MR & $38 \pm 3.2$ & $0.09 \pm 0.01$ & $2.2 \pm 0.6$ \\
& HR & $52 \pm 1.8$ & $0.07 \pm 0.01$ & $2.0 \pm 0.3$ \\
$t_{a}=1.0 \mathrm{~mm}$ & QS & $9 \pm 1.9$ & $0.16 \pm 0.08$ & $0.6 \pm 0.5$ \\
& MR & $35 \pm 3.0$ & $0.10 \pm 0.01$ & $2.2 \pm 0.4$ \\
& HR & $38 \pm 8.4$ & $0.07 \pm 0.01$ & $1.4 \pm 0.2$ \\
\hline
\end{tabular}

and a trapezoidal-shaped curve for the QS and MR loading regimes. The initial elastic response seems to exhibit a rate-dependent behaviour. Similarly to the film adhesive, the mean peak stress for each loading condition remains almost constant independently of the adhesive thickness considering the high deviation of the shape-given parameters $\sigma, \delta$ and $G_{\max }$ - see Table 2. The mean final displacement at fracture increases as the adhesive layer thickens up to a threshold of $t_{\mathrm{a}}=0.5 \mathrm{~mm}$. After this adhesive layer thickness, the displacement at fracture decreases. Both an increase of the mean peak stress and the displacement with larger adhesive thickness lead to an increase of $G_{\max }$. After the aforementioned threshold is reached, the dissipated energy before failure decreases. This behaviour suggests the existence of an optimal adhesive thickness close to $0.5 \mathrm{~mm}$. However, more experiments with different adhesive thickness need to be performed if one wants to find the absolute optimum. 


\section{Discussion}

\subsection{On the rate, thickness, surface treatment, and composition dependency of the adhesive bonds}

Fig. 9 shows the rate dependency of the peak stress $\sigma$ and the resulting dissipated energy $G_{\max }$. For both adhesive types and adhesive thicknesses, $\sigma$ is significantly higher while $\delta$ and $G_{\max }$ are significantly lower under impact than under quasi-static conditions.

In order to understand the effect that the adhesive thickness has on the joint performance, the peak stress and the dissipated energy - which includes the effect of the final failure displacement - are plotted in Fig 10 (a) and (b). Under quasi-static loading conditions, both adhesives show a slight decrease in stress and an increase of failure displacement and dissipated energy as the thickness of the adhesive layer increases.

Under the high-rate regime, the peak stress and the dissipated energy for both adhesives is shown independent of the thickness. In the case of medium-rates, the dissipated energy for the film adhesive increases significantly when the thickness is increased from 0.1 to $0.3 \mathrm{~mm}$, and remains constant for further increase to $0.5 \mathrm{~mm}$. In the case of the paste adhesive, the energy tends to increase proportionally to the thickness up to a value of $0.5 \mathrm{~mm}$ - it then decreases for a thickness of $1.0 \mathrm{~mm}$. It is reasonable to believe that the existence of voids and micro-cracks in the paste adhesive has a higher influence on the mechanical performance under impact when the thickness of the adhesive is limited to $0.1 \mathrm{~mm}$. This could be explained with the small amount of material, where the particles are not able to demonstrate their full support of reinforcement. The similar observation could be observed for the thinnest film adhesive thickness. Generally, it can be demonstrated that the dissipated energy (in other references referred as fracture toughness) increases with increasing adhesive thickness. This is on reasonable agreement with data published previously [16].

Fig 10 (c) and (d) shows the differences in behaviour between both adhesive types when tested under identical conditions. The main differences between both adhesives are found at the microstructure level: (i) the AF 163-2OST film adhesive uses a matrix of rubber particle reinforced epoxy supported by a glass fibre matte and comes as a film, while (ii) the 2216 B/A Gray is a two-component epoxy paste adhesive toughened with Kaolin particles. In general, the film adhesive shows higher values of stress, critical deformation and dissipated energy than the paste adhesive independently of adhesive thickness and loading rate. This difference might be due to the different nature of the toughening elements and epoxy matrix. 
The performance of an adhesive joint does not only depend on the adhesive itself but also on the surface finish of the adherents. Here, three different surface treatments were studied for the paste adhesive $2216 \mathrm{~B} / \mathrm{A}$ Gray using an adhesive layer thickness of $0.1 \mathrm{~mm}$ and quasi-static loading conditions. The three surface treatments of the end-caps are: (i) anodization, (ii) sand blasting, and (iii) plane polishing. Fig. 11 shows the obtained results for the stress and the dissipated energy performance versus the deformation. As expected no surface treatment i.e. surface polished results in a very poor stress and dissipated energy. Sand blasting results in a higher stress and dissipated energy, but still below those obtained using anodised surfaces. Hence, anodization is considered a critical step in the bonding procedure. Moreover, Fig. 11 shows the fracture surface for both plane polished and sand-blasted surface treatments. In both cases, adhesion failure (1) is predominant, however, the area for interface failure of sand-blasted specimens is much smaller than that of fully-polished specimens.

\subsection{On the nature of the failure mechanism: fractography analysis}

In order to prove the homogeneity of the stress over the adhesive interface, an analysis of the fracture surface under different testing conditions is carried out. For this purpose, a scanning electron microscope (SEM) was employed. Representative surfaces for each one of the adhesive types are shown in Fig 12 . In both cases, a predominant cohesive failure - see (2) in Fig 12 - is observed. However, adhesion failure (1) is also visible in those areas closer to the edges of the specimen. In this regard, the adhesive film shows slightly more homogeneous fracture surface than the paste adhesive - the adhesion area of the film is approximately $200 \mu \mathrm{m}$ wide while that of the paste adhesive is approximately $500 \mu \mathrm{m}$ wide - see Fig 12 (b) and (d). Singular stress concentration around the free edge is believed responsible for this small inhomogeneity. Fig 13 shows the influence of the adhesive thickness on the fracture surface. For both adhesive compositions, a narrow area of interface failure next to the specimen free edge can be observed - this is marked as (1) in Fig 13 (a) and (c). As the thickness of the adhesive increases, several layers of fractured adhesive are visible - see areas marked as (2) in Fig 13 (b) and (d). Also, the areas of near-to-interface failure are not observed in the $0.5 \mathrm{~mm}$ adhesives. Through the thicker adhesive, more energy as a consequence of larger deformation is required in order to separate the two bonded surfaces. This results in a larger critical deformation with increasing adhesive thickness and therefore an enlargement of the dissipated energy. 
For a better understanding of the cohesive fracture of each adhesive type, the roughness and the position of the post-failure crack path was measured using a 3D micro coordinate measurement machine (Alicona 3D Profilometer). The profile height of two representative $0.5 \mathrm{~mm}$ thickness adhesive joints tested under MR conditions are shown in Fig 14 . The film adhesive shows a more homogeneous fracture surface than the paste adhesive: the line measurement and the color-map show that the fracture of the film adhesive is approximately half of the total thickness. This is clearly representative of cohesive failure. However, the fracture of the paste adhesive is less homogeneous with larger profile changes and it shows areas which look like adhesion failure. However, the roughness of those areas and the peak right at the edge of the sample seem to indicate a near-tointerface fracture rather than an adhesion fracture - see Fig 14 (d).

Fig 15 shows detailed micrographs of the nature of each adhesive. A main difference is appreciated: the fracture surface of the film adhesive indicates a smooth appearance while the paste adhesive shows a very rough fracture surface - see Fig 15 (a) and (b) respectively. The thin crack lines marked as (2) in Fig 15(a) show the smooth fracture surface. The structure marks as (6) in Fig 15 (b) indicate a very rough fracture of the paste adhesive. Moreover, Fig 15 shows micrographs of the fracture surfaces under both QS and HR conditions. The main failure modes for the adhesive film are shown as numbered areas in Fig.15 (a). When a load is applied at the adhesive joint, the adhesive elongates until the strength of the adhesive is reached. After that, the adhesive deforms plastically until the critical values of the adhesive components are reached. Theoretically, the fibre support should not have a strong influence on the mechanical performance. However, as reported in [42], the fibre scrim may introduce imperfections in the epoxy matrix which would reduce the mechanical performance of the adhesive. With this in mind, it is reasonable to assume that the first cracks will appear in the interface between fibre scrim and matrix. Detail (1) in Fig. 15(a) shows the imprint of a fibre in the matrix - the surface is unstructured, indicating that there was little resistance as the fibre detached from the epoxy (3). In contrast, the fractured epoxy shows strong marks of shear deformation - see detail (2) in Fig. 15(a). Since the adhesive shows certain porosity, cracks within the pores/ voids (4) tend to appear first as the final fracture approaches. This loss in stability is evident in the stress-displacement curve indicates the beginning of the softening slope. After this point, fibre rupture with fibre pull-out (5) is observed. This phenomena may result in an exponential falling slope rather than a linear one - see Fig. 7.

The fracture surface of the paste adhesive is different. It shows a rough and unstructured 
behaviour - see Fig. 15 (b). The applied load lets the paste adhesive deform plastically until the first cracks appear - usually perpendicular to the loading direction in the epoxy matrix, and specially at the interface between particles and matrix. With further loading, these cracks coalesce - see detail (7). Moreover, the limited bonding at the interface between epoxy and particle is shown in detail (8). The process of fracture of each adhesive is illustrated in Fig. 15.

In Fig. 15 one cannot find a noticeable difference between QS and HR, this suggests that an important part of the mechanical performance of the adhesives is dictated by the epoxy adhesive itself. The adhesive is a polymer which consists of molecule chains, thus, one may expect that the mechanical behaviour is based heavily on the independent behaviour of these chains. When a load is applied rapidly, the entangled molecule chains are not able to reorient in order to adjust themselves to the direction of applied load. This results in a higher resistance but a lower displacement-tofailure when the adhesives are tested under high-rate. In contrast, if the load is applied slowly over a long period of time, the molecule chains have time to reorient. In this case, the adhesive is capable to use its full mechanical properties until the maximum values are reached [43].

\subsection{Validity, advantages and limitations of the proposed methodology to measure cohesion}

In order to prove the validity of the methodology, a standard DCB test has been simulated and compared to experimental results found in the literature [44. The simulation employs cohesive elements which have been assigned a traction separation law which matches the behaviour measured experimentally. The procedure sequence is shown in Fig. 16 (a). The numerical simulation of the experimental investigation has been performed using the finite element code Abaqus/Implicit. The geometry of the finite element model of the DCB has been generated accordingly to the literature [44. The adherents were modelled using 8-node single integration point hexahedral elements with the properties of a typical aluminium alloy 2024- $\mathrm{T}$ Alclad $E=66 \mathrm{GPa}, \sigma_{y}=350 \mathrm{MPa}$ and $\nu=$ 0.33 , 44. The adhesive layer with a bondline thickness of $t^{a}=0.2 \mathrm{~mm}$ was modelled using cohesive elements in combination with the simplified tri-linear traction separation law extracted from the experiments. A pre-crack of $15 \mathrm{~mm}$ was introduced as described in [44. The specimen was then pulled at a constant velocity of $v=0.2 \mathrm{~mm} / \mathrm{min}$. The simulated results are in good agreement with the experimental force-displacement curve - see Fig. 16 (b). Therefore, one may assume that the traction separation curve as directly measured in the experiments is a good representation of the cohesive response of the adhesive interface. 
The proposed specimen geometry and experimental methodology have a few advantages for the characterisation of adhesive interfaces when compared to standard DCB tests. For example: (i) the same setup and geometry is used independently of rate, thus, the rate-dependent behaviour can be directly compared, (ii) the high-rate results are absent of dynamic effects which introduce noise in the measurements - as opposed to DCB experimentation, and (iii) the traction-separation can be measured directly, there is no need to use analytical models. In this last regard, Fig. 17 compares the measured values for the dissipated energy to the ones obtained in literature using DCB tests. One can see that the values of dissipated energy derived from DCB tests fall within the trend measured using our approach. The deviation may be due to the influence of the surface treatment which plays a crucial role in adhesively bonded joints. Nevertheless, the proposed experimental methodology also has some disadvantages: (i) the measurement requires a series of specialised equipment - such as several high-speed cameras for DIC - that are not widely available. Also, for QS and MR experiments, the synchronisation procedure can be challenging - if the synchronisation is not exact, the calculated values of dissipated energy may be inaccurate.

\section{Analytical model for adhesive behaviour: a rate-dependent traction-separation law}

The experimental campaign for characterising the adhesive joint behaviour under quasi-static, medium-rate and high-rate loading conditions enables the development of a phenomenological material model of the adhesive behaviour. The experimental stress-displacement curves and the derivation of the dissipated energy are used to generate a trapezoidal shaped traction separation law (TSL) - Fig. 18. This is a valid approach when considering the adhesive layer to be an interface zone.

The damage evolution process takes place in terms of the degradation of the adhesive stiffness. This can be described by the scalar damage variable $d$. The traction component of the material after damage initiation is described following

$$
t=(1-d) K \delta
$$

where $t$ is the traction, $\delta$ the separation value and $K$ is the stiffness, which is calculated using the 
Young's modulus and the adhesive layer thickness

$$
K=\frac{E}{t_{\mathrm{a}}} .
$$

Due to the higher stiffness of the adherents, the Poisson's contraction of the adhesive may be restricted which could affect the measurement of the adhesive interface stiffness $K$, [45]. The criteria for damage initiation is written in terms of separation as $\delta_{0}$ and follows

$$
\delta_{0}=\frac{\sigma_{\max }}{K}
$$

where $\sigma_{\max }$ is the maximum traction at initiation. The separation at the end of the plateau of the plastic region, $\delta_{\mathrm{pl}}$, is given by

$$
\delta_{\mathrm{pl}}=\delta_{0}+\frac{\alpha \cdot G_{\max }}{\sigma_{\max }}
$$

where $G_{\max }$ is the dissipated energy, and as it is introduced by [46] $\alpha$ is the ratio of the plastic energy and the dissipated energy, following the rate dependent equation

$$
\alpha=\alpha_{0} \cdot\left(\frac{\dot{\delta}}{\dot{\delta}_{0}}\right)^{q}
$$

Since the whole area under the traction-separation curve corresponds to the obtained dissipated energy, the final separation $\delta_{\mathrm{f}}$ can be obtained using

$$
\delta_{\mathrm{f}}=\delta_{0}-\delta_{\mathrm{pl}}+\frac{2 \cdot G_{\max }}{\sigma_{\max }}
$$

where $\delta_{\mathrm{pl}}$ is the end of the plastic plateau. The separation rate dependency of the initial traction is described using a power-law as

$$
\sigma_{\max }=\sigma_{0} \cdot\left(\frac{\dot{\delta}}{\dot{\delta}_{0}}\right)^{m}
$$

and for the dissipated energy following

$$
G_{\max }=G_{0} \cdot\left(\frac{\dot{\delta}}{\dot{\delta_{0}}}\right)^{b}
$$


where $\sigma_{0}, G_{0}$ and $\dot{\delta}_{0}$ are the reference values of stress, dissipated energy and separation rate respectively. The parameters $m$ and $b$ are the separation rate sensitivity exponents for the stress and the dissipated energy respectively.

Then, the damage variable $d$ can be fully defined as follows

$$
d=\left\{\begin{array}{cc}
0 & , \delta \leq \delta_{0} \\
1-\frac{\delta_{0}}{\delta} & , \delta_{0}<\delta \leq \delta_{p l} \\
1-\frac{\delta_{0}\left(\delta_{f}-\delta\right)}{\delta\left(\delta_{f}-\delta_{p l}\right)} & , \delta_{p l}<\delta \leq \delta_{f} \\
1 & , \delta>\delta_{f}
\end{array}\right.
$$

\subsection{Determination of the TSL parameters}

The experimental results shown in Figs 7 were used to identify the parameters which describe the behaviour of the model. Experimentally obtained values of the peak stress, plastic separation and final failure separation for each thickness and loading rate were used to calibrate the relevant rate sensitivity parameters $(\mathrm{m}, \mathrm{b}, \mathrm{q})$ and the reference parameter $\left(\sigma_{0}, G_{0}, \alpha_{0}\right)$ for stress, dissipated energy and plastic ratio. With the least square method, optimal parameters for the analytical model were generated using an optimisation tool. The reference separation rate parameters $\dot{\delta}_{0}$ is assumed to be unity in order to keep the units consistent.

The identified parameters are shown in Table 3 for the film and the paste adhesive respectively. The results from the analytical model are compared to the experiments in Fig. 19. The model is able to capture the thickness-dependent and rate-dependent behaviour of the joint; the tractionseparation behaviour is in good agreement with the experimental results. However, for a paste adhesive the results do not correlate as good as for the film adhesive. High experimental scatter is believed to be influencing the obtained constants. The TSL law can be implemented directly into the cohesive zone models commonly available in many of the commercial finite element analysis packages to simulate industrially relevant bonded components.

\section{Conclusions}

The rate dependent behaviour for mode I deformation and failure of a thermosetting epoxy film adhesive and a two-component paste adhesive has been characterised experimentally. A novel 
Table 3: Calibration of TSL parameters for both adhesives.

\begin{tabular}{c|ccccccc}
\hline Parameters & $T_{0}(\mathrm{MPa})$ & $\mathrm{m}$ & $G_{0}\left(\frac{N}{\mathrm{~mm}}\right)$ & $\mathrm{b}$ & $\alpha_{0}(-)$ & $\mathrm{q}$ & $\mathrm{E}(\mathrm{MPa})$ \\
\hline AF 163-2OST & 41.57 & 0.034 & 4.92 & -0.101 & 0.455 & -0.050 & 2000 \\
2216 B/A Gray & 19.25 & 0.112 & 2.00 & 0.001 & 0.591 & -0.024 & 2500 \\
\hline
\end{tabular}

experimental methodology for the characterisation of the mechanical performance of adhesive joints has been developed for this purpose. The results show the influence of loading rate, adhesive composition and adhesive thickness on the mechanical properties and are used to propose a ratedependent analytical traction-separation model. The following specific conclusions can be drawn:

1. A methodology able to capture both the reversible and irreversible deformation of the adhesive under three-different loading regimes is presented. It relies upon the stress wave propagation and the combination of both ultra-high-speed-photography and medium-to-low-speedphotography based DIC. The optimal setup and specimen design was derived using numerical analyses - this was used to ensure accurate property measurement and traction homogeniety within the interface. Post-mortem fractography proved this approach successful.

2. Metal-to-metal adhesive joints using two different commercially available adhesives - a film and a paste adhesive - were characterised using the developed methods. Their mechanical properties show strong dependence on the adhesive thickness, the adhesive architecture, the surface treatment and the loading rate.

3. Particularly, the strength tends to increase proportionally to the loading rate and the dissipated energy tends to decrease proportionally to the loading rate. This trend occurs independently of adhesive morphology and adhesive thickness. The effect of adhesive thickness is different - the maximum strength is shown independent, but the dissipated energy is larger for thicker interfaces. The loss of volume of the thinner adhesives is believed responsible: the size of voids and other imperfections are close to the total thickness thus a representative volume element is not achieved.

4. The film adhesive showed higher strength and dissipated energy than the paste adhesive. Highresolution fractography was used to elucidate this difference in behaviour: (i) AF 163-2OST shows intrinsic damage: matrix fracture, crack across the voids, fibre rapture, debonding of the fibres from the matrix and fibre pull-out; (ii) 2216 B/A Gray shows a rougher surface influenced by the appearance of micro-cracks and debonding of Kaolin particles from the 
matrix.

5. A rate-dependent traction-separation law is proposed. The model is able to capture the ratedependency of both the maximum stress and the dissipated energy accurately. This is suitable for implementation as a cohesive zone model which can then be used to assess numerically the mechanical performance of adhesively-bonded components under practical conditions.

\section{Acknowledgements}

The authors of this paper are grateful to Dr. Bamber Blackman and Dr. Rodolfo Rito, Imperial College London, for preparing the film-adhesive joints. Furthermore, the authors are grateful to Dr. Francisca Martinez Hergueta for her support and suggestions. The authors are grateful to Jeffrey Fullerton and Stuart Carter, Impact Engineering Laboratory, University of Oxford, for their assistance in the manufacturing of the specimens. The authors acknowledge the funding from Rolls-Royce plc which enabled this investigation. 


\section{References}

[1] A. J. Kinloch, M. S. G. Little, J. F. Watts, The role of the interface in the environmental failure of adhesive joints, Acta Materialia 48 (18-19) (2000) 4543-4553. doi:10.1016/ S1359-6454(00) 00240-8.

[2] M. D. Banea, L. F. M. da Silva, Adhesively bonded joints in composite materials: an overview, Journal of materials Design and Applications 223 (1) (2009) 1-18. doi:10.1243/14644207.

[3] J. D. Russell, 3.3 The Impact of Large Integrated and Bonded Composite Structures on Future Military Transport Aircraft, in: P. W. R. Beaumont, C. H. Zweben (Eds.), Comprehensive Composite Materials $\{\mathrm{II}\}$, Elsevier, 2018, pp. 91-130. doi:https://doi.org/10.1016/ B978-0-12-803581-8.09925-2.

URL https://www.sciencedirect.com/science/article/pii/B9780128035818099252

[4] S. Li, M. D. Thouless, A. M. Waas, J. A. Schroeder, P. D. Zavattieri, Competing failure mechanisms in mixed-mode fracture of an adhesively bonded polymer-matrix composite, International Journal of Adhesion and Adhesives 26 (8) (2006) 609-616. doi:10.1016/j.ijadhadh. 2005.07 .010 .

[5] M. Y. Tsai, J. Morton, An investigation into the stresses in double-lap adhesive joints with laminated composite adherends, International Journal of Solids and Structures 47 (24) (2010) 3317-3325. doi:10.1016/j.ijsolstr.2010.08.011.

URL http://dx.doi.org/10.1016/j.ijsolstr.2010.08.011

[6] M. May, G. Ganzenmüller, J. Wolfrum, S. Heimbs, Analysis of composite T-joint designs for enhanced resistance to hydrodynamic ram, Composite Structures 125 (2015) 188-194. doi: 10.1016/j.compstruct.2015.02.012. URL http://dx.doi.org/10.1016/j.compstruct.2015.02.012

[7] I. A. Ashcroft, D. J. Hughes, S. J. Shaw, Mode I fracture of epoxy bonded composite joints: 1. Quasi-static loading, International Journal of Adhesion and Adhesives 21 (2) (2001) 87-89. doi:10.1016/S0143-7496(00)00038-5.

[8] Q. D. Yang, M. D. Thouless, S. M. Ward, Elastic-plastic mode-II fracture of adhesive joints, 
International Journal of Solids and Structures 38 (18) (2001) 3251-3262. doi:10.1016/ S0020-7683(00) 00221-3.

[9] J. R. Reeder, J. R. Crews Jr., Mixed-mode bending method for delamination testing, AIAA Journal 28 (7) (1990) 1270-1276. doi:10.2514/3.25204.

URL http://arc.aiaa.org/doi/10.2514/3.25204

[10] U. T. Carvalho, R. D. Campilho, Application of the direct method for cohesive law estimation applied to the strength prediction of double-lap joints, Theoretical and Applied Fracture Mechanics 85 (2016) 140-148. doi:10.1016/j.tafmec.2016.08.018.

URL http://dx.doi.org/10.1016/j.tafmec.2016.08.018

[11] R. Fernandes, J. A. Chousal, M. F. de Moura, J. Xavier, Determination of cohesive laws of composite bonded joints under mode II loading, Composite Structures 106 (2013) 646-652. doi:10.1016/j.compstruct.2013.07.027.

URL http://dx.doi.org/10.1016/j.compositesb.2013.04.007

[12] H. Cui, S. Koussios, Y. Li, A. Beukers, Constitutive law of adhesive layer measured with mixed mode bending test, Engineering Fracture Mechanics 127 (2014) 235-251. doi:10.1016/j. engfracmech.2014.06.011.

URL http://dx.doi.org/10.1016/j.engfracmech.2014.06.011

[13] M. May, O. Hesebeck, S. Marzi, W. Böhme, J. Lienhard, S. Kilchert, M. Brede, S. Hiermaier, Rate dependent behavior of crash-optimized adhesives Experimental characterization, model development, and simulation, Engineering Fracture Mechanics 133 (2015) 112-137. doi:10. 1016/j.engfracmech.2014.11.006.

URL http://linkinghub.elsevier.com/retrieve/pii/S0013794414003804

[14] J. J. M. Machado, E. A. S. Marques, L. F. M. da Silva, Adhesives and adhesive joints under impact loadings: An overview, The Journal of Adhesion 0 (0) (2017) 1-32. doi:10.1080/ 00218464.2017 .1282349 .

URL https://doi.org/10.1080/00218464.2017.1282349

[15] C. Sarrado, A. Turon, J. Costa, J. Renart, An experimental analysis of the fracture behavior of composite bonded joints in terms of cohesive laws, Composites Part A: Applied Science and 
Manufacturing 90 (2016) 234-242. doi:10.1016/j.compositesa.2016.07.004.

URL http://dx.doi.org/10.1016/j.compositesa.2016.07.004

[16] C. Sarrado, A. Turon, J. Costa, J. Renart, On the validity of linear elastic fracture mechanics methods to measure the fracture toughness of adhesive joints, International Journal of Solids and Structures 81 (2015) 110-116. doi:10.1016/j.ijsolstr.2015.11.016.

[17] B. R. K. Blackman, A. J. Kinloch, F. S. Rodriguez Sanchez, W. S. Teo, J. G. Williams, The fracture behaviour of structural adhesives under high rates of testing, Engineering Fracture Mechanics 76 (18) (2009) 2868-2889. doi:10.1016/j.engfracmech.2009.07.013.

URL http://linkinghub.elsevier.com/retrieve/pii/S0013794409002380

[18] F. Kadioglu, R. Adams, Flexible adhesives for automotive application under impact loading, International Journal of Adhesion and Adhesives (2014) 1-6doi:10.1016/j.ijadhadh.2014. 08.001

URL http://linkinghub.elsevier .com/retrieve/pii/S0143749614001699

[19] J. Casas-Rodriguez, I. Ashcroft, V. Silberschmidt, Damage evolution in adhesive joints subjected to impact fatigue, Journal of Sound and Vibration 308 (3-5) (2007) 467-478. doi: $10.1016 / j \cdot j s v .2007 .03 .088$ URL http://linkinghub.elsevier .com/retrieve/pii/S0022460X07002994

[20] W. Hufenbach, F. M. Ibraim, A. Langkamp, R. Böhm, A. Hornig, Charpy impact tests on composite structures - An experimental and numerical investigation, Composites Science and Technology 68 (12) (2008) 2391-2400. doi:10.1016/j.compscitech.2007.10.008.

URL http://linkinghub.elsevier.com/retrieve/pii/S0266353807004071

[21] P. Kumar, S. Tiwari, R. Singh, Characterization of toughened bonded interface against fracture and impact loads, International Journal of Adhesion and Adhesives 25 (6) (2005) 527-533. doi:10.1016/j.ijadhadh.2005.01.007

URL http://linkinghub.elsevier.com/retrieve/pii/S0143749605000291

[22] K. Ravi-Chandar, Dynamic Fracture, first edit Edition, Vol. 1, Elsevier Ltd, 2004.

[23] T. Yokoyama, K. Nakai, Determination of the impact tensile strength of structural adhesive butt joints with a modified split Hopkinson pressure bar, International Journal of Adhesion 
and Adhesives (2014) 1-11doi:10.1016/j.ijadhadh.2014.07.011.

URL http://linkinghub.elsevier.com/retrieve/pii/S014374961400150X

[24] T. Yokoyama, Experimental determination of impact tensile properties of adhesive butt joints with the split Hopkinson bar, The Journal of Strain Analysis for Engineering Design 38 (3) (2003) 233-245. doi:10.1243/030932403765310563.

URL http://journals.pepublishing.com/openurl.asp?genre=article $\{\&\}$ id=doi: $10.1243 / 030932403765310563$

[25] H. Kolsky, An Investigation of the Mechanical Properties of Materials at very High Rates of Loading, Proceedings of the Physical Society.Section B 62 (11) (1949) 676-700.

URL http: //stacks . iop.org/0370-1301/62/i=11/a=302

[26] C. Keisler, J. Lataillade, The effect of Substrate Roughness Characteristics on Wettability and on the Mechanical Properties of Adhesive Joints Loaded at High Strain Rates, Journal of Adhesion Science and Technology 9 (1995) 395-411.

[27] O. Sen, S. A. Tekalur, C. Jilek, The determination of dynamic strength of single lap joints using the split Hopkinson pressure bar, International Journal of Adhesion and Adhesives 31 (6) (2011) 541-549. doi:10.1016/j.ijadhadh.2011.04.006. URL http://linkinghub.elsevier . com/retrieve/pii/S0143749611000558

[28] T. Yokoyama, H. Shimizu, Evaluation of Impact Shear Strength of Adhesive Joints with the Split Hopkinson Bar, JSME International Journal 41 (4) (1998) 503-509.

[29] H. Zhao, X. Duan, M. Ma, L. Lu, Z. Cai, P. C. Wang, J. D. Fickes, Dynamic characteristics of adhesive bonded high strength steel joints, Science and Technology of Welding and Joining 15 (6) (2010) 486-490. doi:10.1179/136217110x12714217309731.

[30] A. Gilat, T. E. Schmidt, A. L. Walker, Full field strain measurement in compression and tensile split Hopkinson bar experiments, Experimental Mechanics 49 (2) (2009) 291-302. doi: $10.1007 / \mathrm{s} 11340-008-9157-\mathrm{x}$.

[31] Y. Li, K. T. Ramesh, An optical technique for measurement of material properties in the tension Kolsky bar, International Journal of Impact Engineering 34 (4) (2007) 784-798. doi: 10.1016/j.ijimpeng.2005.12.002 
[32] L. Goglio, L. Peroni, M. Peroni, M. Rossetto, High strain-rate compression and tension behaviour of an epoxy bi-component adhesive, International Journal of Adhesion and Adhesives 28 (7) (2008) 329-339. doi:10.1016/j.ijadhadh.2007.08.004.

[33] T. Iwamoto, T. Nagai, T. Sawa, Experimental and computational investigations on strain rate sensitivity and deformation behavior of bulk materials made of epoxy resin structural adhesive, International Journal of Solids and Structures 47 (2) (2010) 175-185. doi:10.1016/ j.ijsolstr.2009.09.026.

URL http://dx.doi.org/10.1016/j.ijsolstr.2009.09.026

[34] J. Neumayer, P. Kuhn, H. Koerber, R. Hinterhölzl, Experimental Determination of the Tensile and Shear Behaviour of Adhesives under Impact Loading, The Journal of Adhesion 92 (7-9) (2015) 503-516. doi:10.1080/00218464.2015.1092387.

URL http://www. scopus.com/inward/record.url?eid=2-s2. 0-84962339117\{\&\}partnerID=tZOtx3y1

[35] A. Needleman, A continuum Model for Void Nucleation by Inclusion Debonding, Journal of Applied Mechanics 54 (3) (1987) 525-531.

[36] T. Ungsuwarungsri, W. G. Knauss, The role of damage-softened material behavior in the fracture of composites and adhesives, International Journal of Fracture 35 (3) (1987) 221-241. doi:10.1007/BF00015590.

[37] V. Tvergaard, J. W. Hutchinson, The relation between crack growth resistance and fracture process parameters in elastic-plastic solids, Journal of the Mechanics and Physics of Solids 40 (6) (1992) 1377-1397. doi:10.1016/0022-5096(92)90020-3.

[38] M. May, O. Hesebeck, Assessment of experimental methods for calibrating rate-dependent cohesive zone models for predicting failure in adhesively bonded metallic structures, Engineering Failure Analysisdoi:10.1016/j.engfailanal.2014.12.008.

URL http://wWw .sciencedirect.com/science/article/pii/S1350630714003719

[39] H. Kolsky, Stress Waves in Solids, Sound and Vibration 1 (1964) 88-110.

[40] R. Gerlach, C. Kettenbeil, N. Petrinic, A new split Hopkinson tensile bar design, International 
Journal of Impact Engineering 50 (2012) 63-67. doi:10.1016/j.ijimpeng.2012.08.004.

URL http://linkinghub.elsevier .com/retrieve/pii/S0734743X1200156X

[41] F. De Cola, A. Pellegrino, C. Glößner, D. Penumadu, N. Petrinic, Effect of Particle Morphology, Compaction, and Confinement on the High Strain Rate Behavior of Sand, Experimental Mechanicsdoi:10.1007/s11340-017-0331-x.

URL http://link.springer.com/10.1007/s11340-017-0331-x

[42] D. Alvarez Feito, Fracture Mechanics of Carbon Fibre Reinforced Plastics to Ti-alloy Adhesive Joints, Ph.D. thesis, Imperial College London (2012).

[43] M. C. Boyce, D. M. Parks, A. S. Argon, Large inelastic deformation of glassy polymers. part I: rate dependent constitutive model, Mechanics of Materials 7 (1) (1988) 15-33. doi:10.1016/ 0167-6636(88)90003-8.

[44] M. P. Palmares, Strength of Hybrid Laminates Aluminium Carbon-Fibre Joints with Different Lay-up Configurations, Ph.D. thesis, Universidade do Porto (2016).

[45] O. Hesebeck, Consideration of the restriction of lateral contraction in the elastic behaviour of cohesive zone models, International Journal of Adhesion and Adhesives 62 (2015) 165-171. arXiv:arXiv:1511.02061v1, doi:10.1016/j.ijadhadh.2015.07.003. URL http://linkinghub.elsevier.com/retrieve/pii/S0143749615001049

[46] M. May, H. Voß, S. Hiermaier, Predictive modeling of damage and failure in adhesively bonded metallic joints using cohesive interface elements, International Journal of Adhesion and Adhesives 49 (2014) 7-17. doi:10.1016/j.ijadhadh.2013.12.001.

URL http://www.sciencedirect.com/science/article/pii/S014374961300211X 
a) Bonding fixture

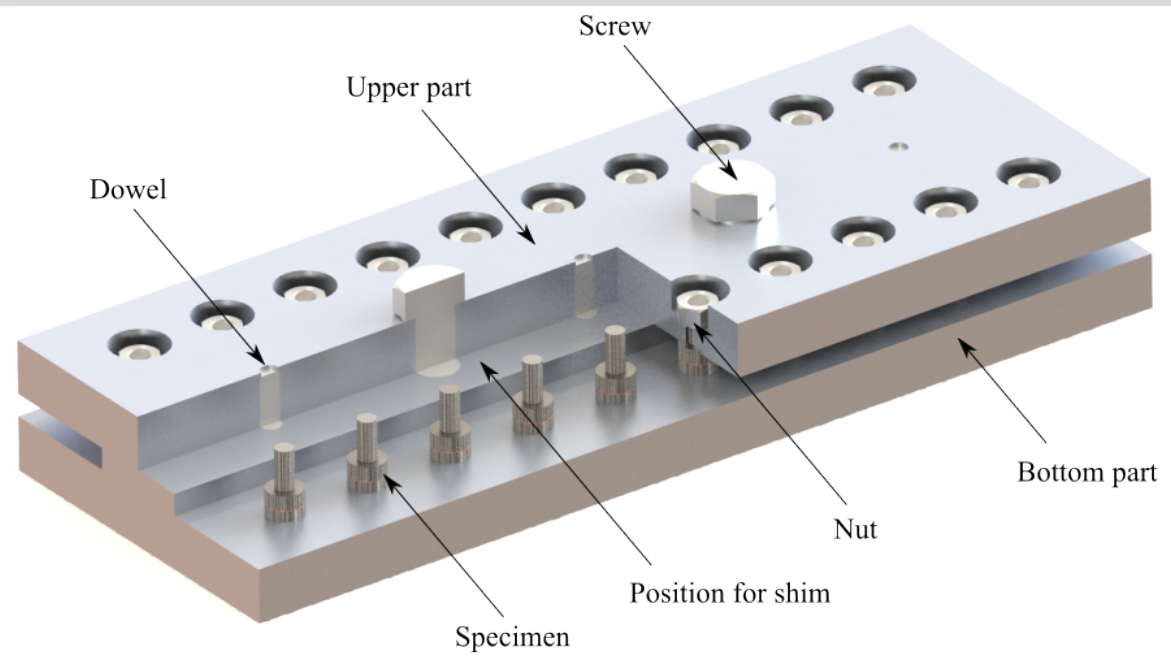

b) Dimensions Mode I specimen

(1) Specimen dimensions

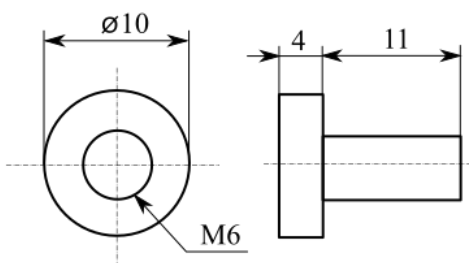

(2) Specimen manufactured

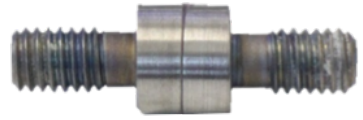

(3) Speckled specimen

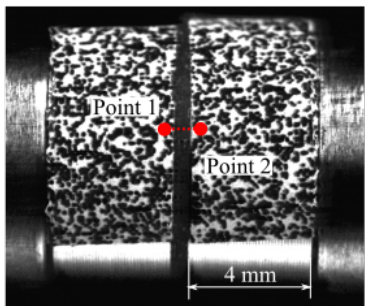

c) Specimen qualification

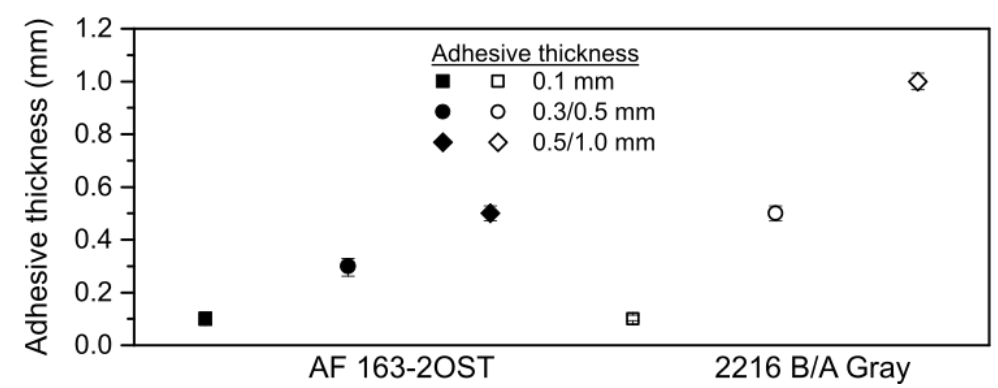

Figure 1: Overview of manufactured tools: a) bonding fixture, b) dimension, design and speckle application of specimen for mode I loading direction and c) bondline thickness qualification. 
a) Split Hopkinson Tensile Bar
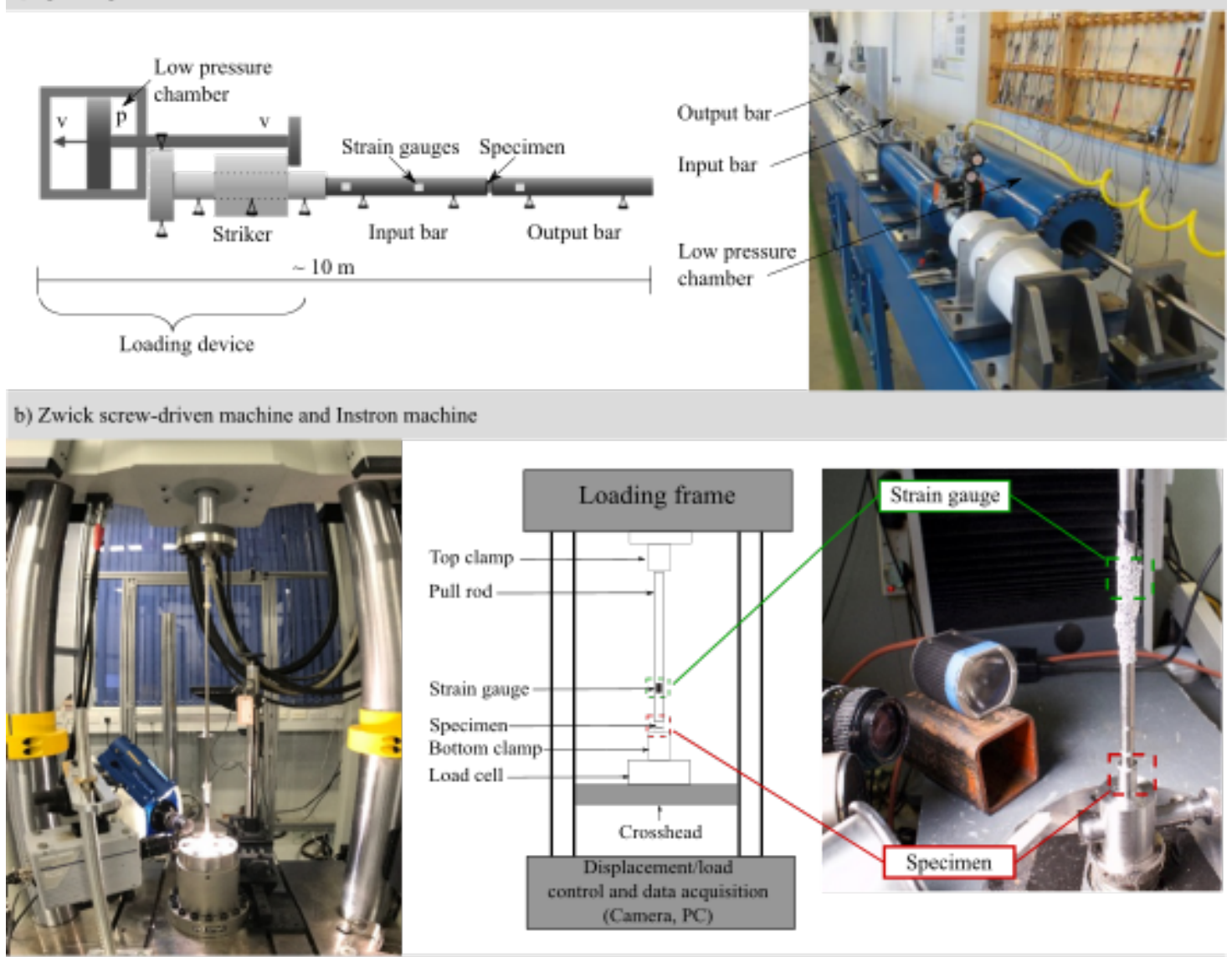

c) Test setup with data acquisition

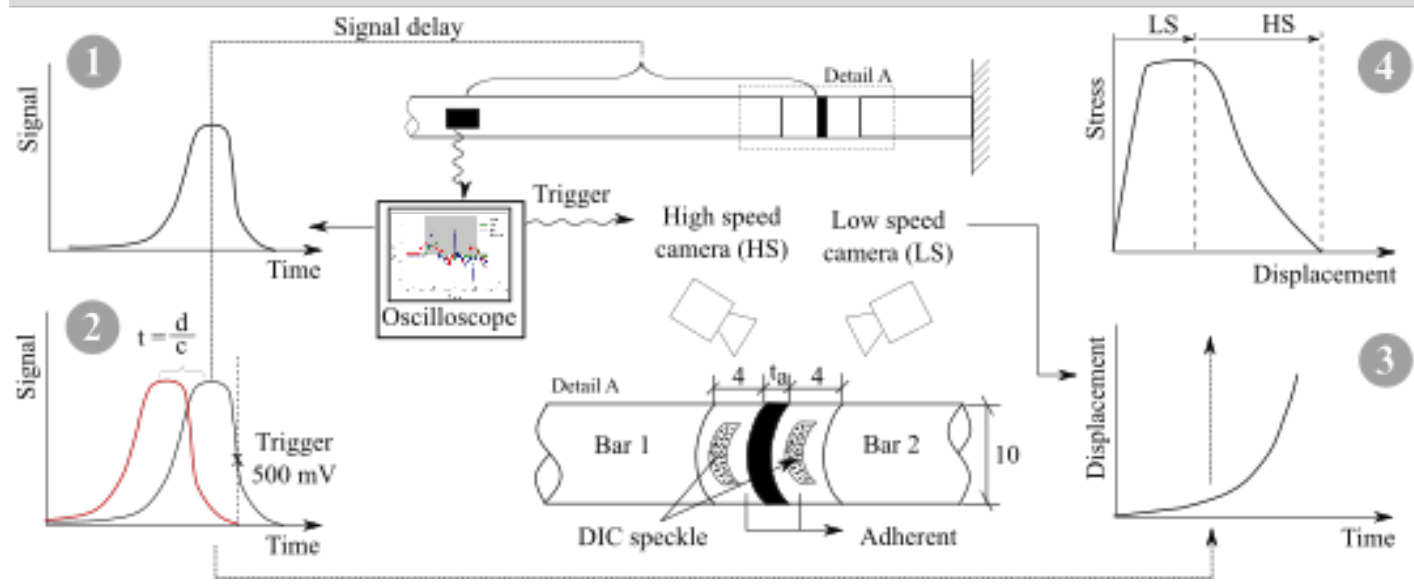

Figure 2: Split Hopkinson Tensile Bar testing apparatus: (a) schematics of the working principle and gas chamber of the experimental SHB. (b) Quasi-static and medium rate setup employing a long loading bar with a strain gauge to capture the softening slope.(c) Data acquisition generating stress-displacement graphs. 

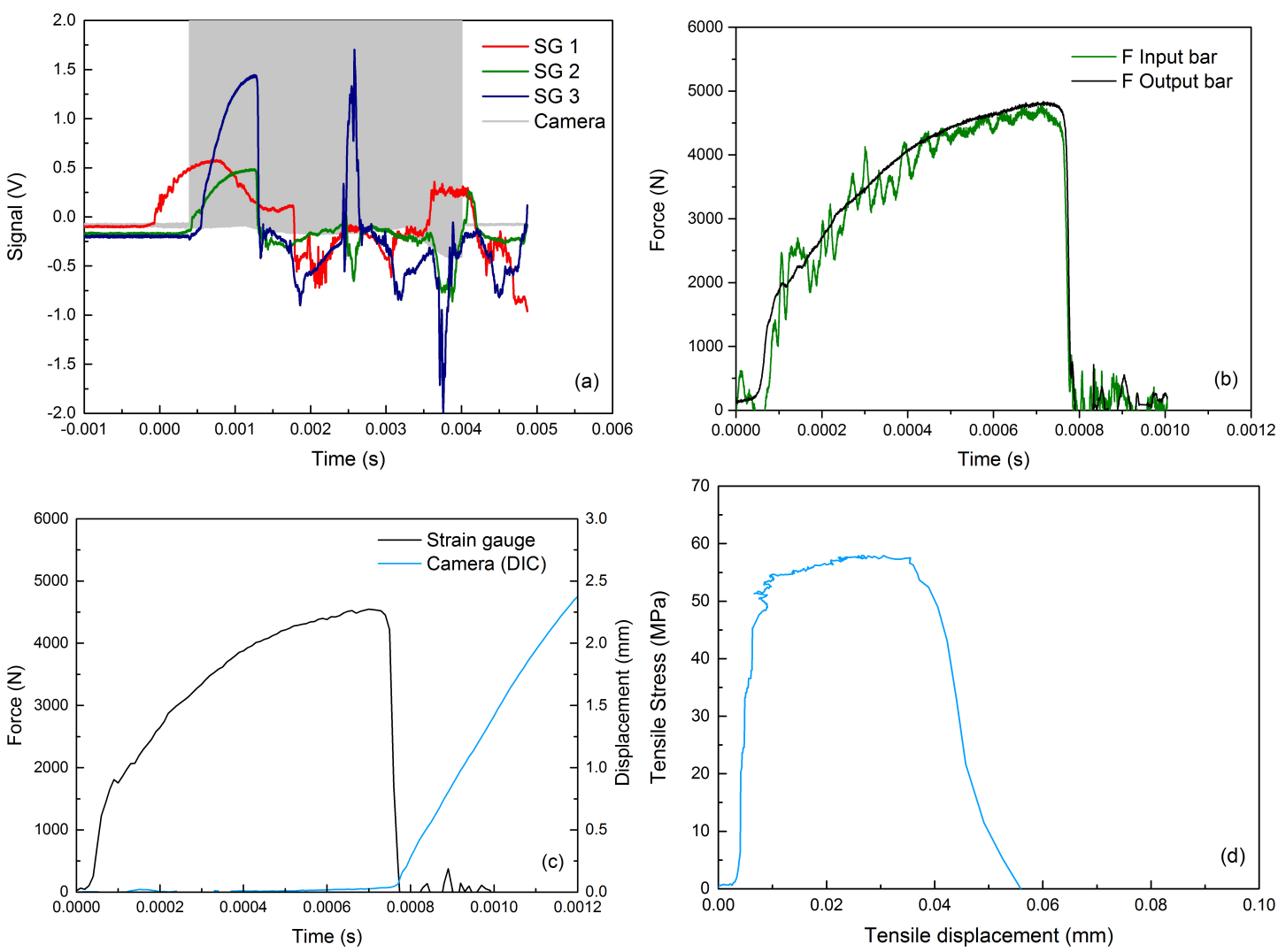

Figure 3: (a) Signal history of each strain gauge and camera trigger, (b) dynamic force equilibrium in the Split Hopkinson Bar, (c) synchronisation of force and displacement and (d) resultant stress-displacement diagram. 

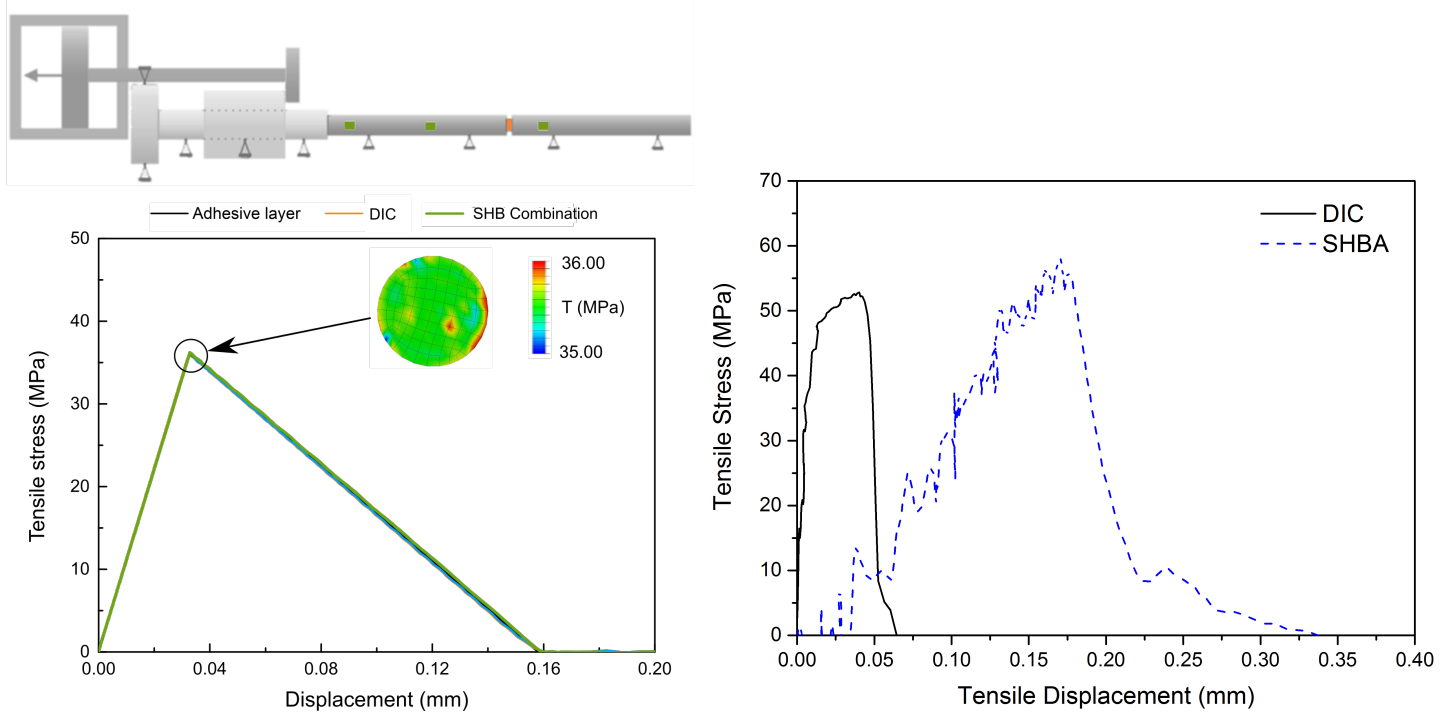

Figure 4: (a) Verification of the experimental HR setup: demonstration of traction homogeneity with the specimen's cross section and (b) comparison of the analysis method SHBA vs. DIC.

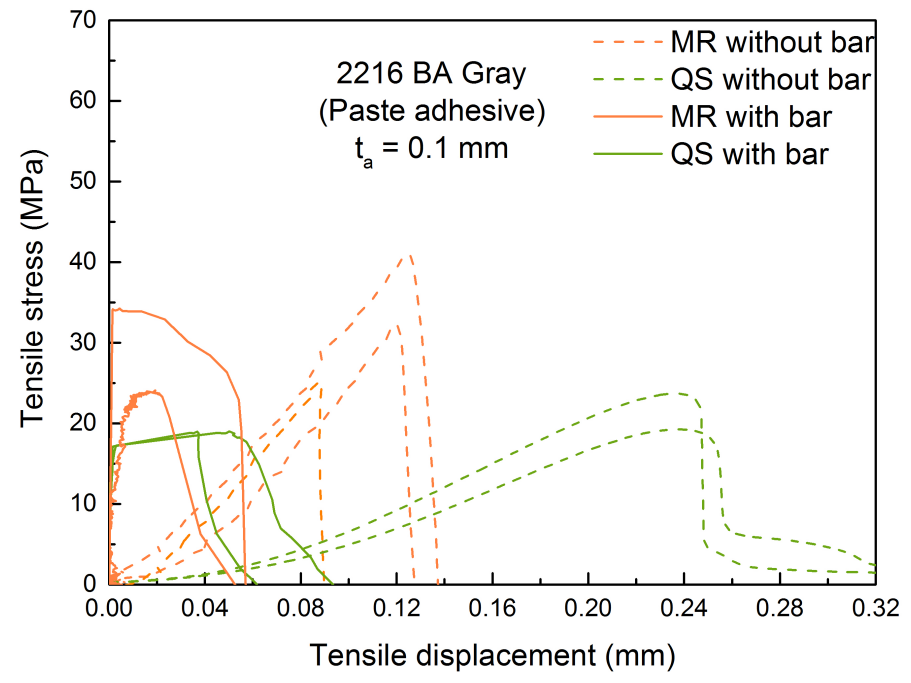

Figure 5: Experimental results without and with long bars in the medium-rate (MR) and quasi-static (QS) loading regimes for a paste adhesive joint with a thickness $t_{\mathrm{a}}=0.1 \mathrm{~mm}$. 


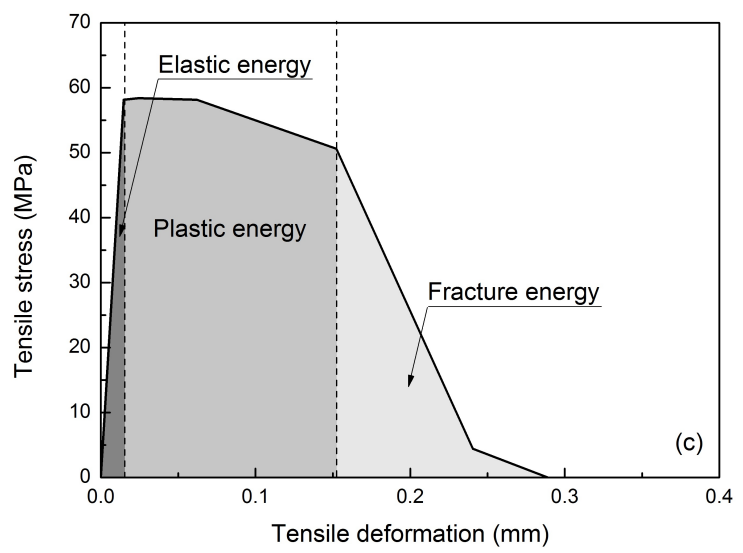

Figure 6: Typical tensile stress-deformation curve showing the approximated areas of energies. 

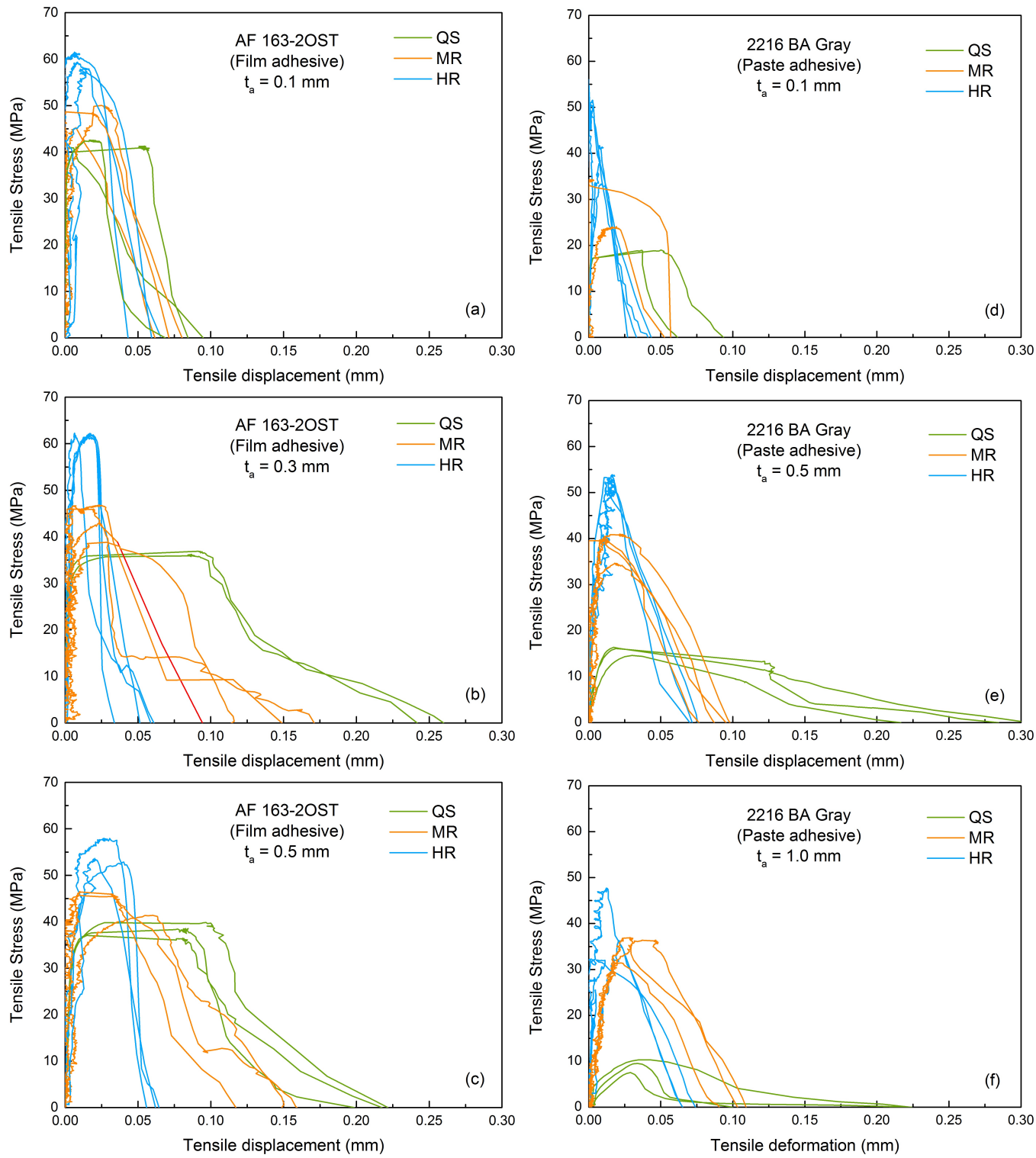

Figure 7: Tensile test results for titanium-to-titanium adhesive joints for the film AF 163-2OST (left-hand side) and paste $2216 \mathrm{~B} / \mathrm{A}$ Gray (right-hand side) adhesives under quasi-static (green), medium-rate (orange) and high-rate (blue) loading conditions for adhesive thickness (a,d) $t_{\mathrm{a}}=0.1 \mathrm{~mm}$, (b) $t_{\mathrm{a}}=0.3 \mathrm{~mm},(\mathrm{c}, \mathrm{e}) t_{\mathrm{a}}=0.5 \mathrm{~mm}$ and (f) $t_{\mathrm{a}}$ $=1.0 \mathrm{~mm}$ 

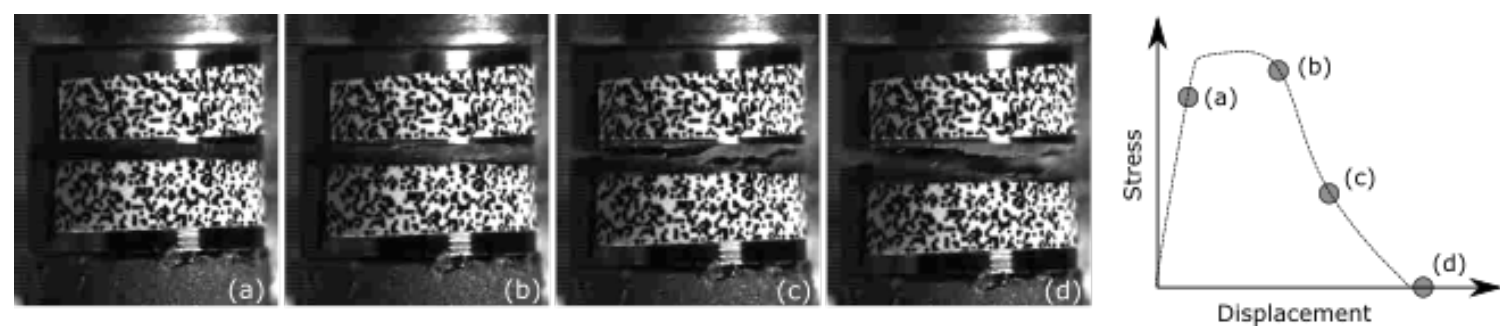

Figure 8: Fracture process of a film-based adhesive joint captured using a high-speed camera in order to generate the kind of stress-displacement curves shown in Fig. 7 The different stages are: (a) Elastic stage, (b) onset of failure, (c) crack propagation and (d) final failure.
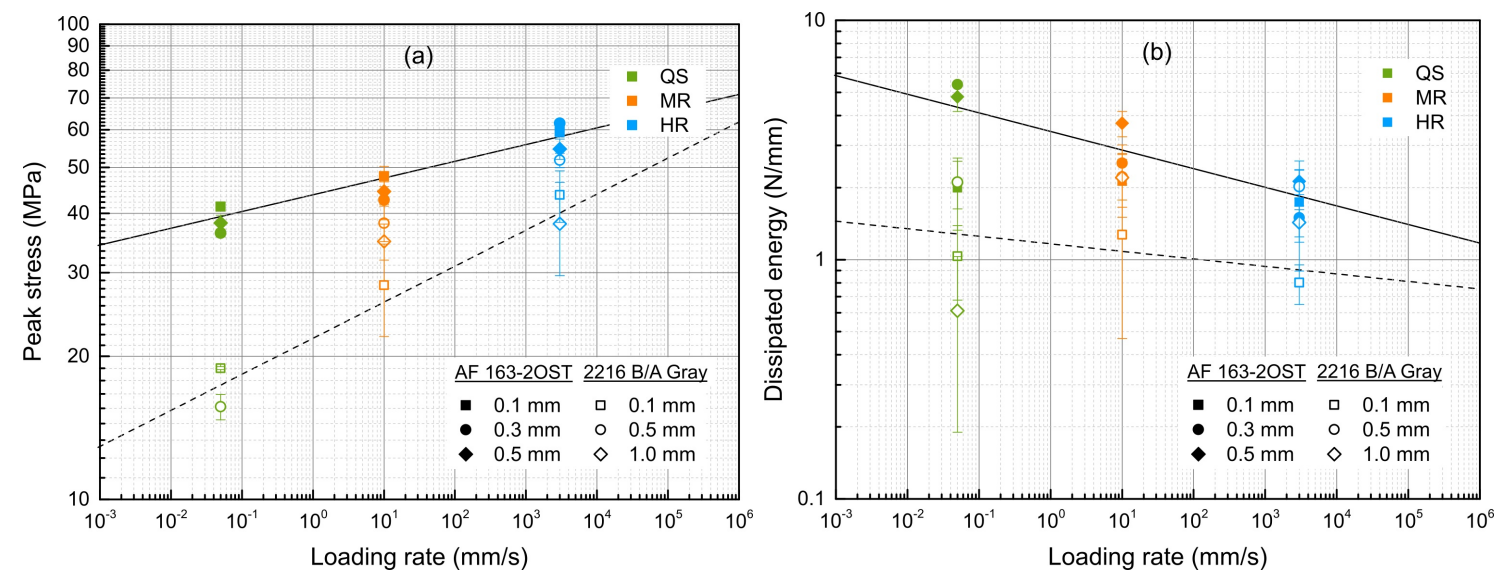

Figure 9: Influence of the loading rate on the: (a) peak stress and (b) dissipated energy for both adhesives. The solid and dotted lines indicating the trend of the rate dependent behaviour of the peak stress and the dissipated energy. 


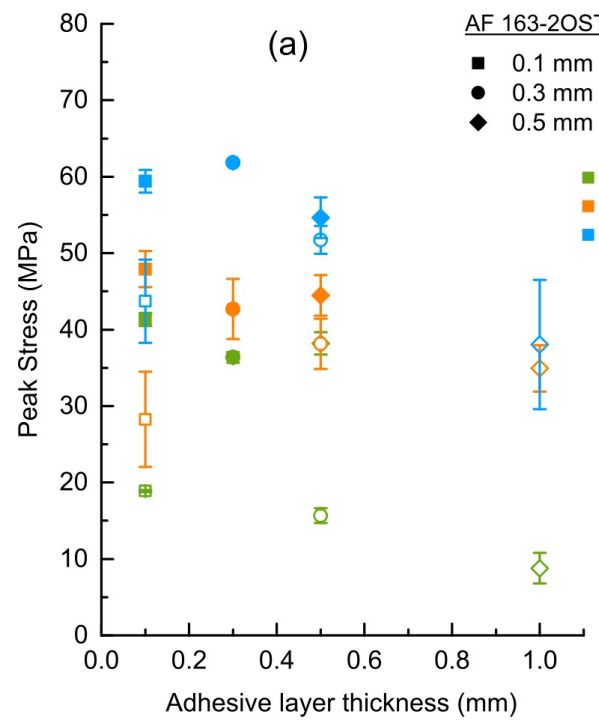

AF 163-20ST 2216 B/A Gray

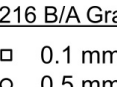

(b)

(b)
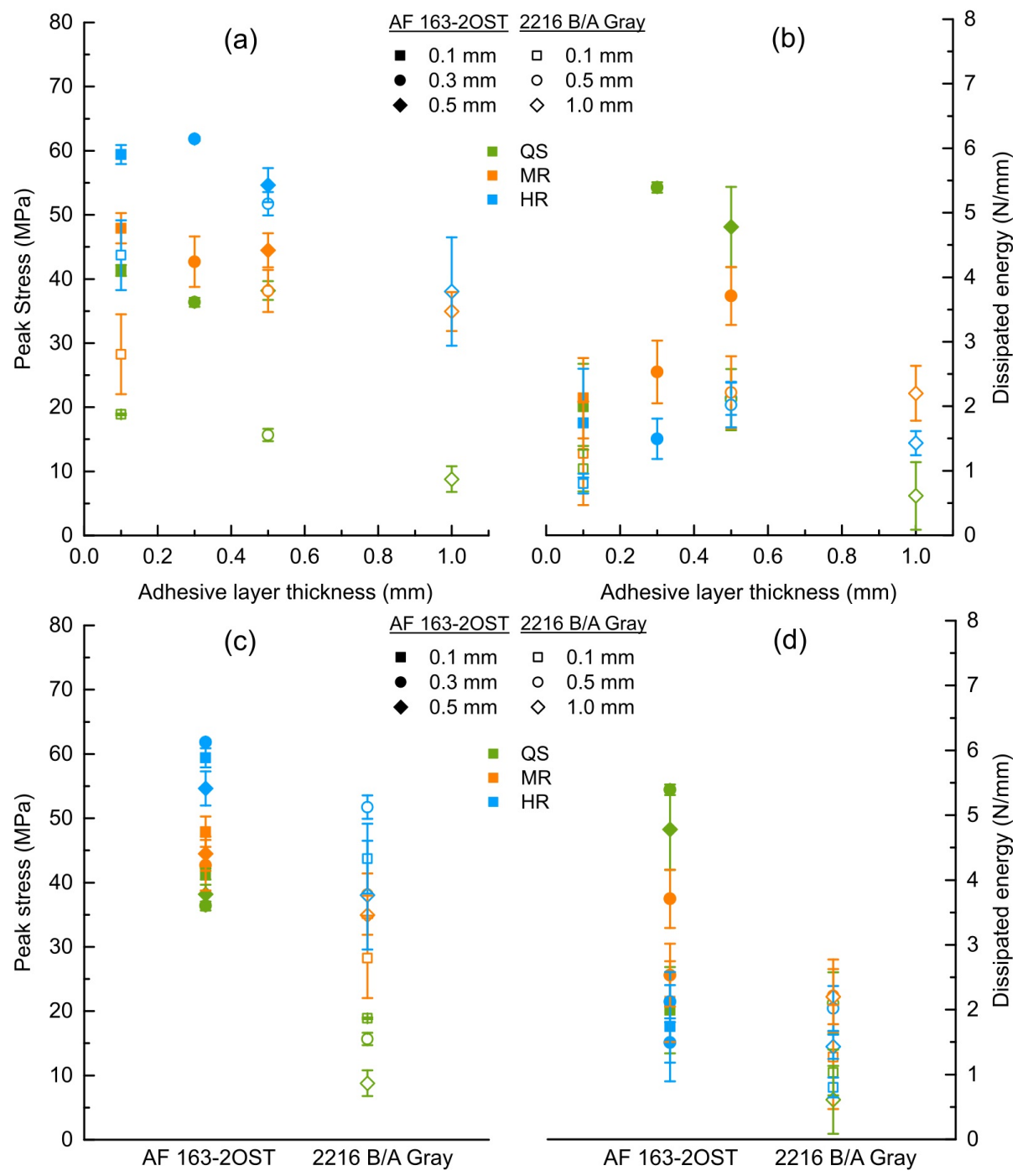

Figure 10: Influence on the tensile stress and dissipated energy of adhesive thickness (a,b) and adhesive composition (c,d). 


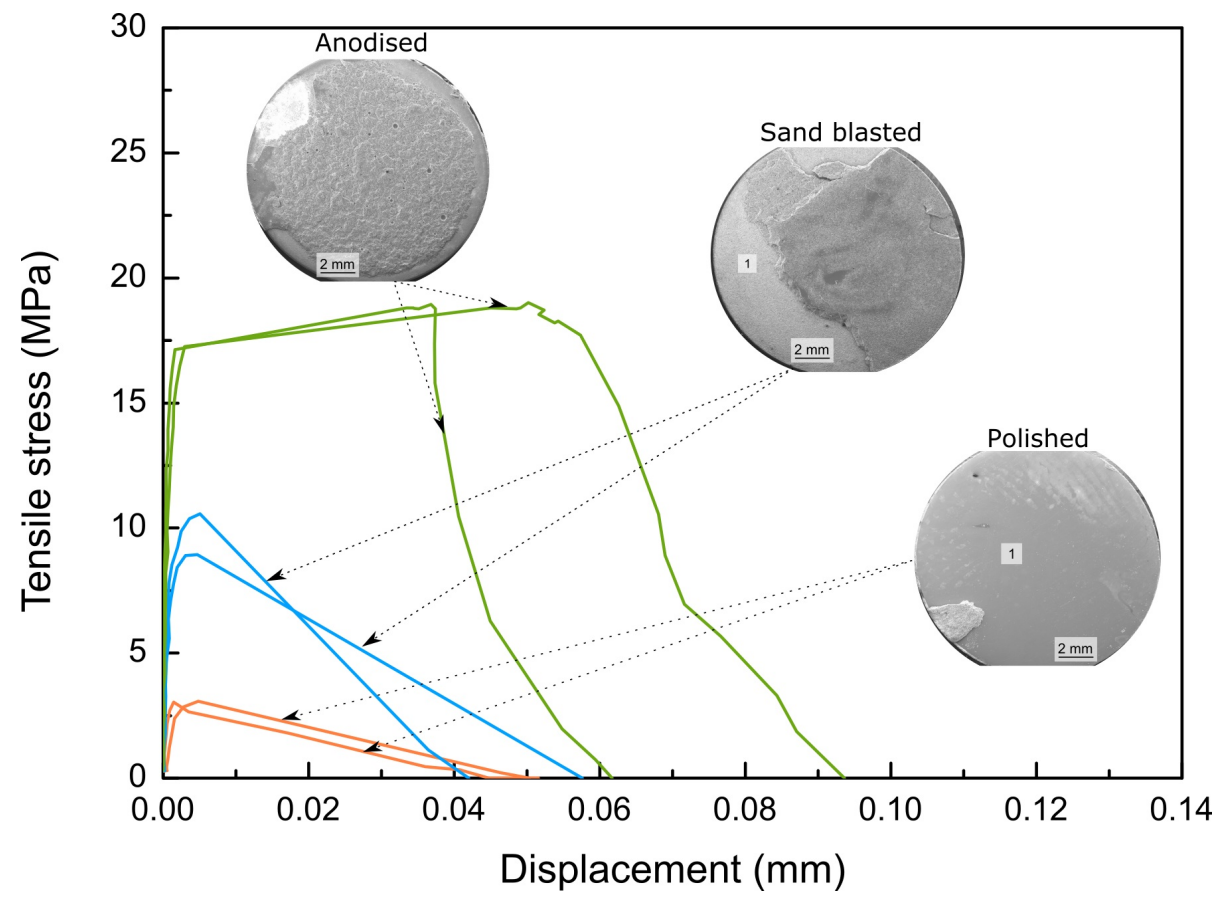

Figure 11: Quasi-static tensile test results showing the tensile stress versus tensile deformation for bonded joints of 2216 B/A Gray with different adherent surface finishes and an adhesive thickness of $0.1 \mathrm{~mm}$. Number (1) identifies the metallic substrate. 

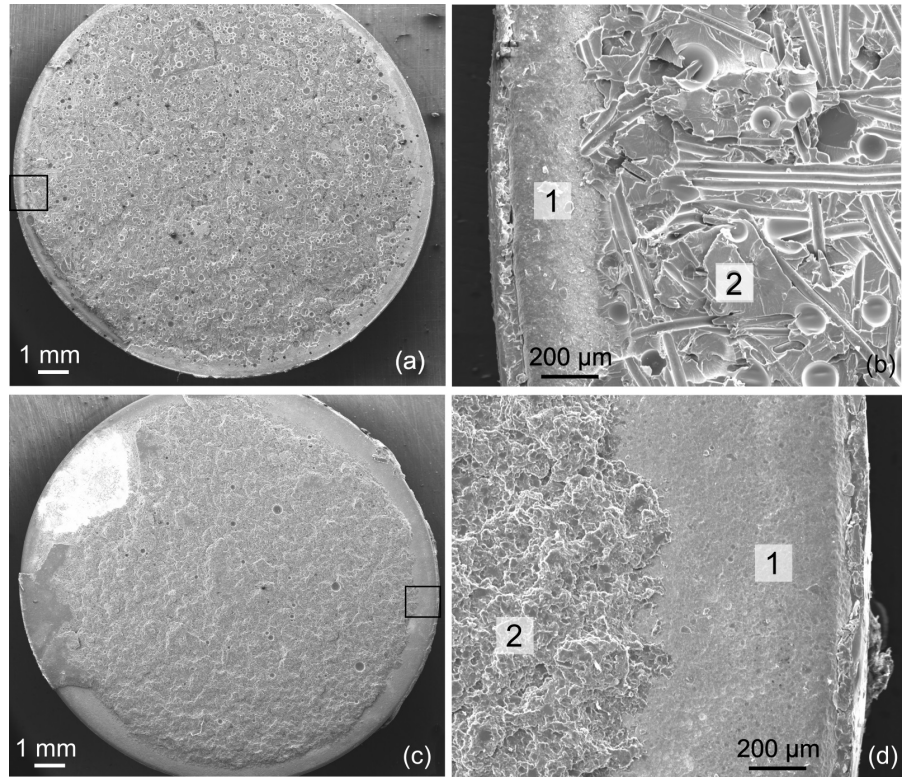

Figure 12: Fracture surfaces of adhesive joint with an adhesive thickness of $t_{a}=0.5 \mathrm{~mm}$ for $(\mathrm{a}, \mathrm{b})$ the AF 163-2OST adhesive and (c,d) the 2216 B/A Gray adhesive. Area marked as (2) represents cohesive failure. Area marked as (1) indicates adhesion failure.
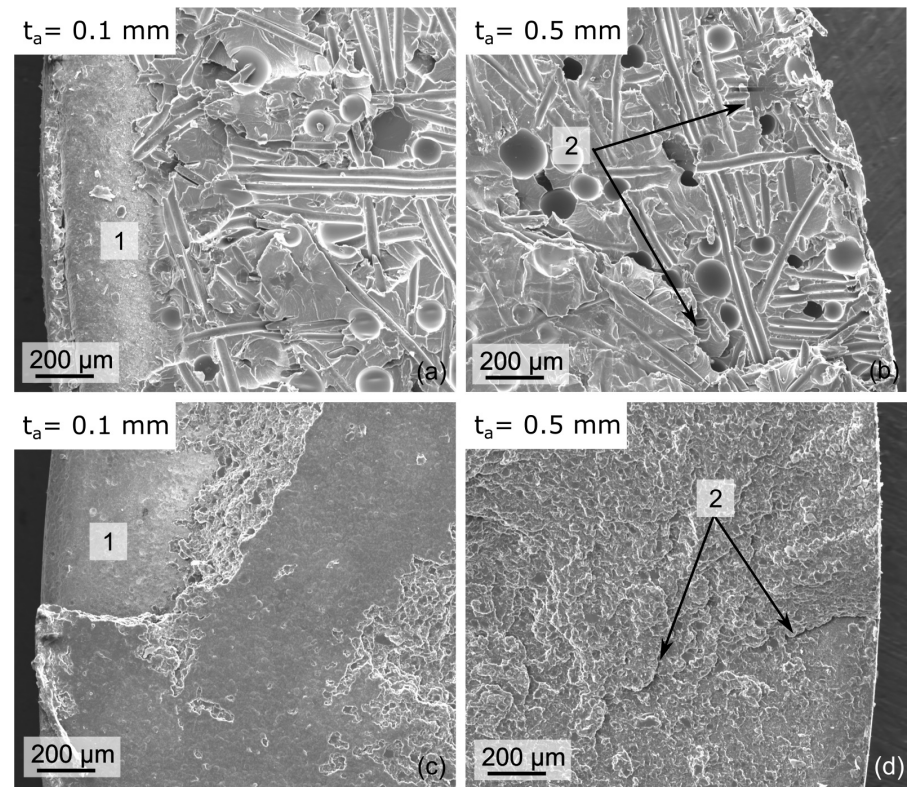

Figure 13: Fracture surface micrographs illustrating the influence of adhesive thickness on the fracture behaviour for $(\mathrm{a}, \mathrm{b}) \mathrm{AF} 163-2 \mathrm{OST}$ and $(\mathrm{c}, \mathrm{d}) 2216 \mathrm{~B} / \mathrm{A}$ Gray with a thickness of $0.1 \mathrm{~mm}(\mathrm{a}, \mathrm{c})$ and $0.5 \mathrm{~mm}(\mathrm{~b}, \mathrm{~d})$ when tested under impact. 

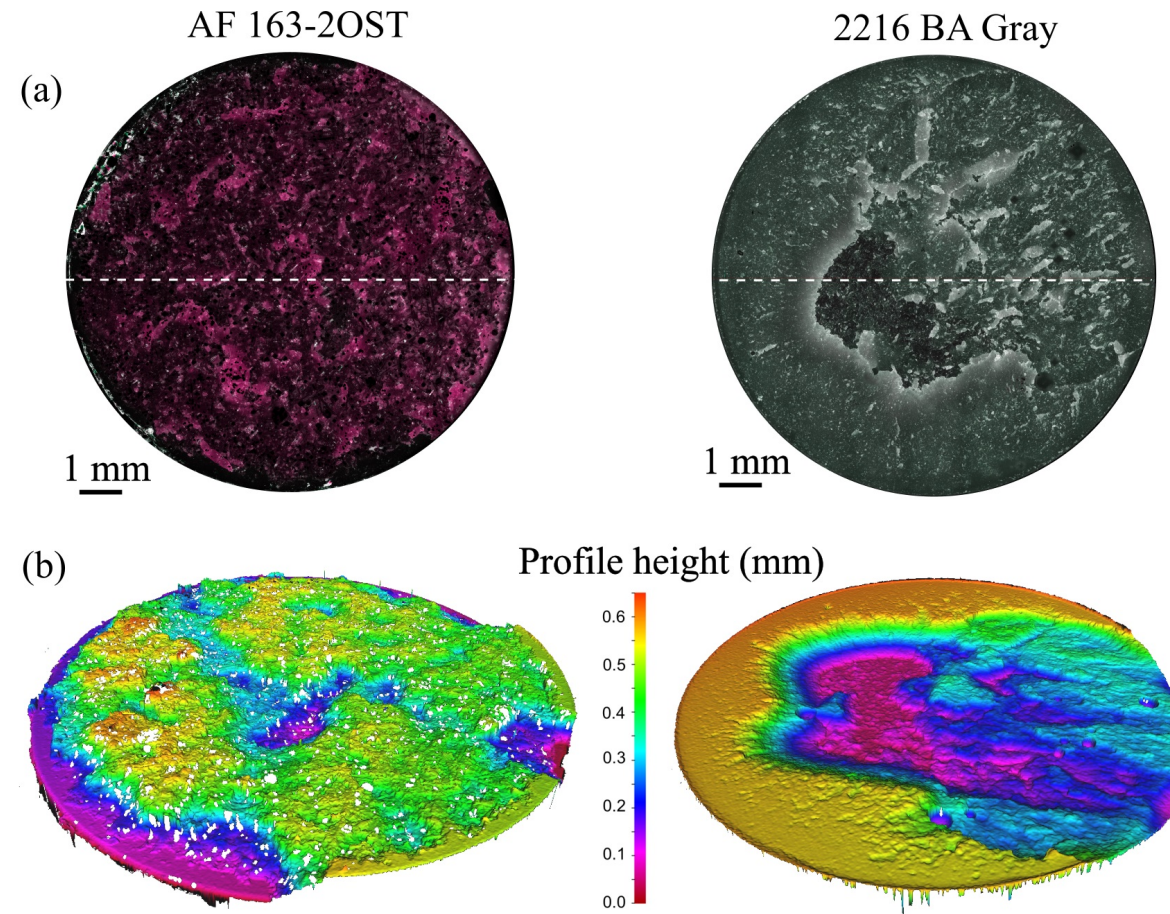

(c)

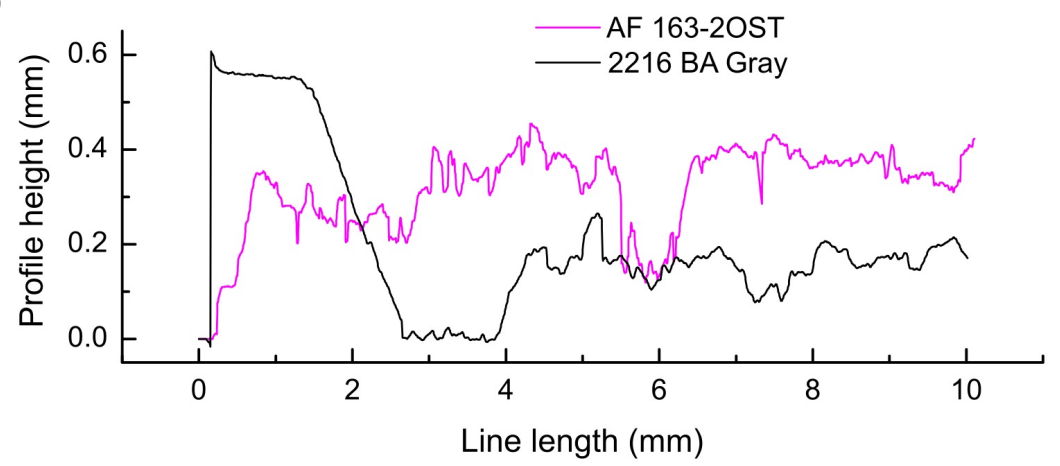

(d)

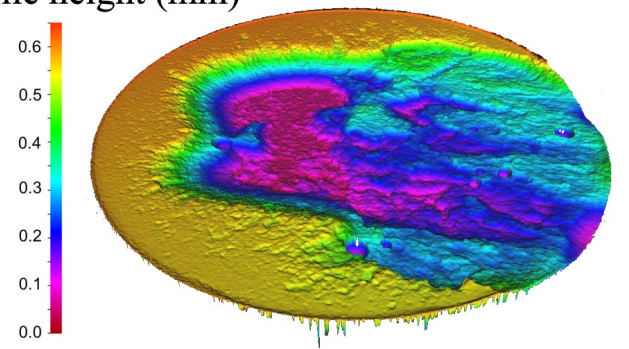

AF 163-2OST

(1) Cohesive fracture

(2) Cohesive near the interface fracture

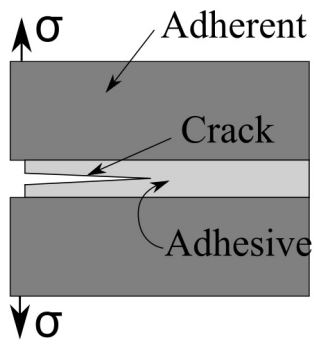

A

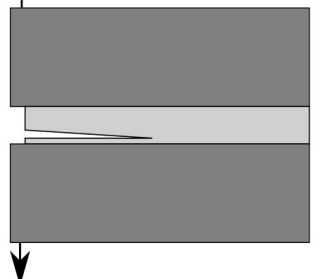

(3) Adhesion or interfacial fracture

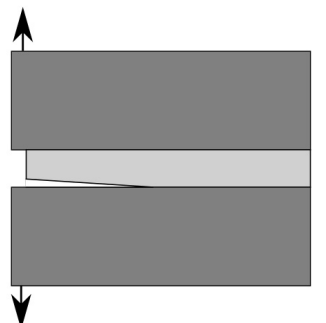

Figure 14: High-detail optical micrographs illustrating the adhesive composition for (a) the adhesives, (b) graphical illustration of the profile for both adhesives, (c) a graph showing the profile height over the diameter and (d) fracture modes of adhesive joints. 
(a) Film adhesive
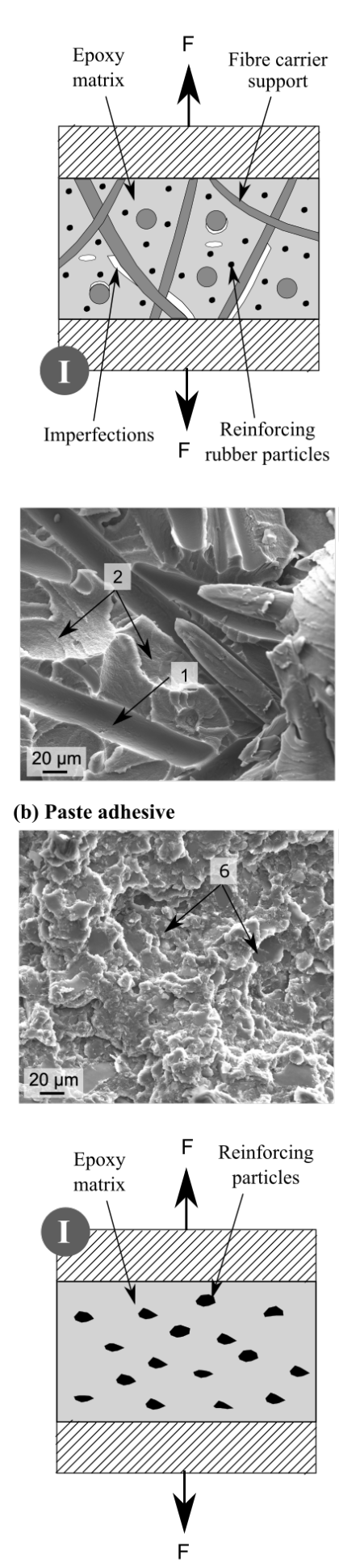
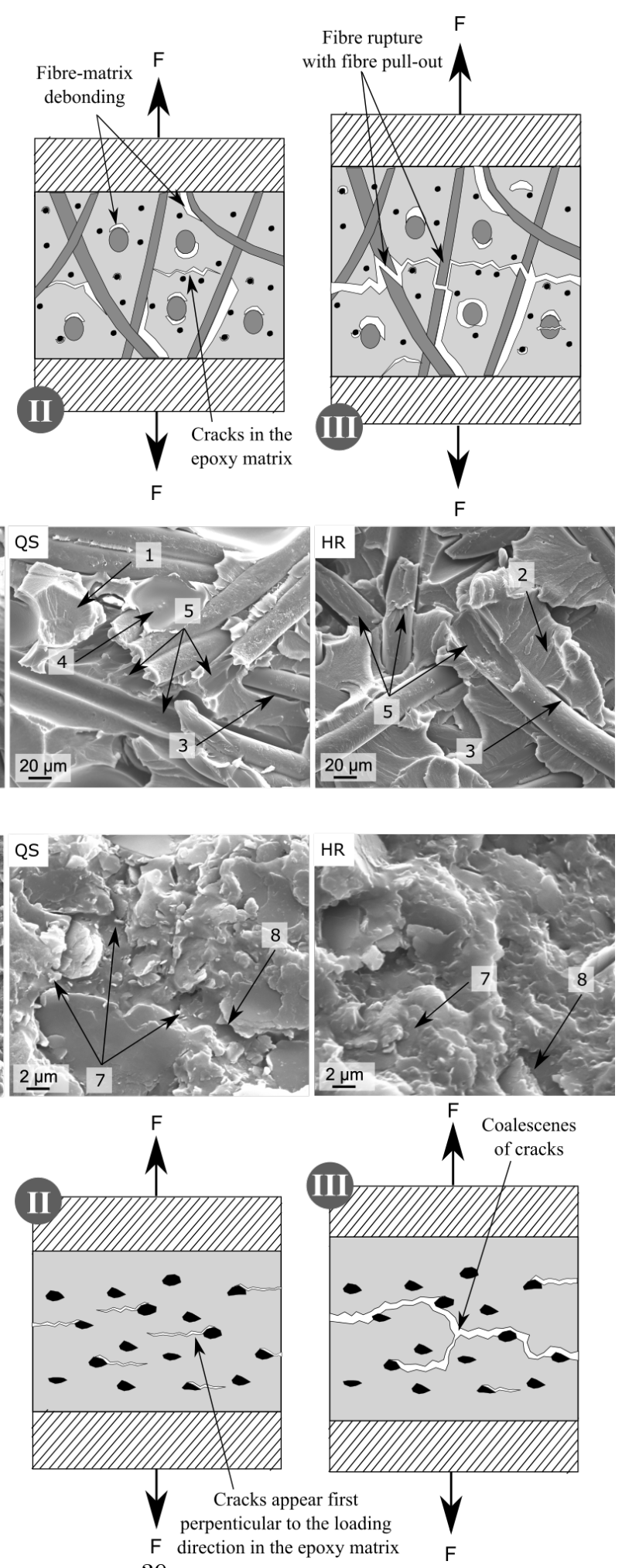

39

Figure 15: Fracture surface micrographs showing detailed features of the film adhesive AF 163-2OST (a) and paste adhesive 2216 B/A Gray (b) when tested under quasi-static conditions and high-rate conditions including the fracture process for each adhesive 
a) Validation procedure

(1) New experimental methodology

(2) Standardised configuration comparison

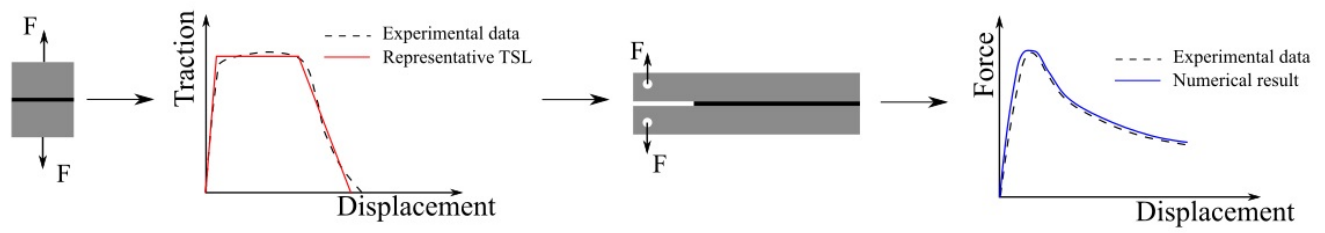

b) Result of the validation process

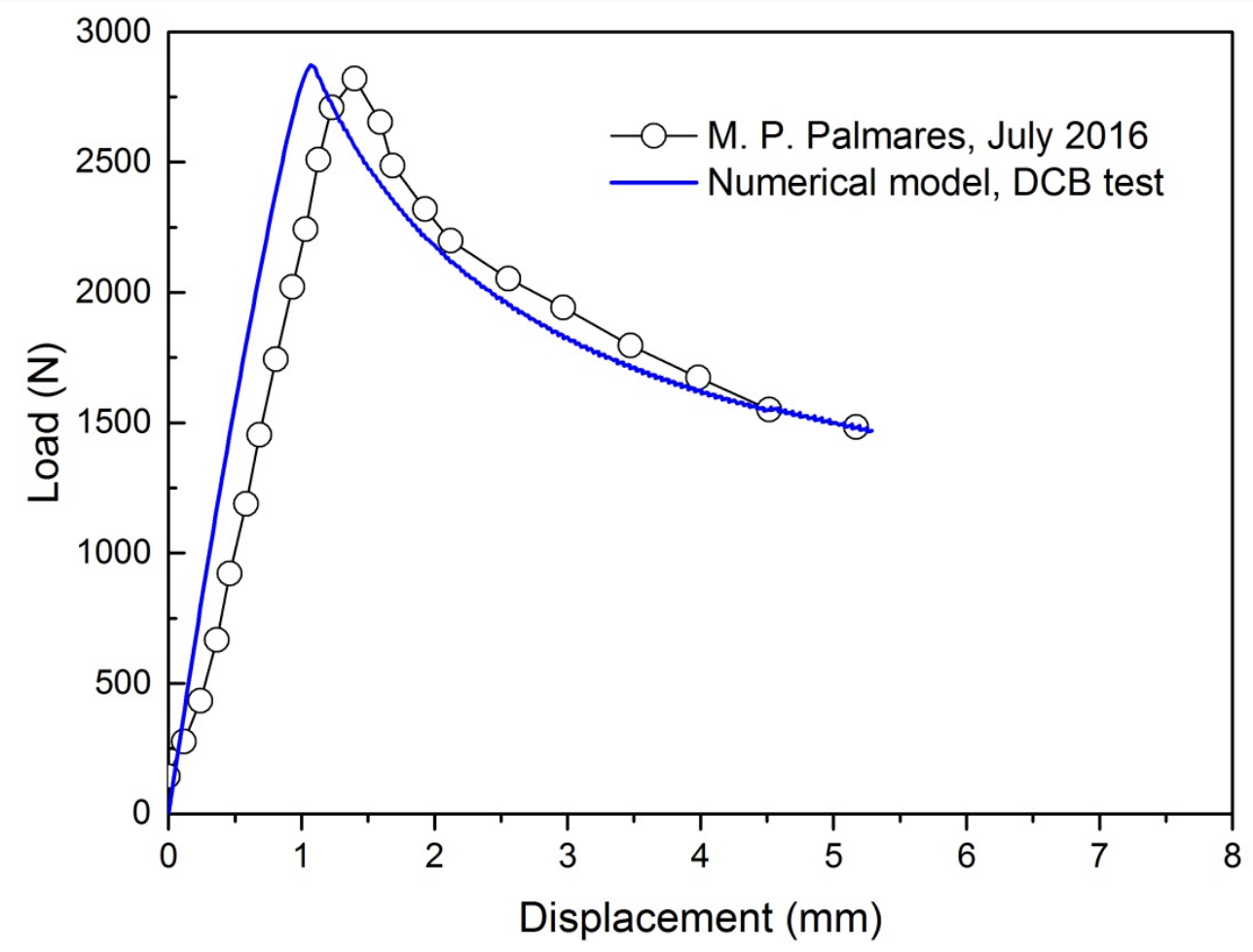

Figure 16: Demonstration of a) the validation sequence and b) the comparison of results obtained from literature and simulation. 

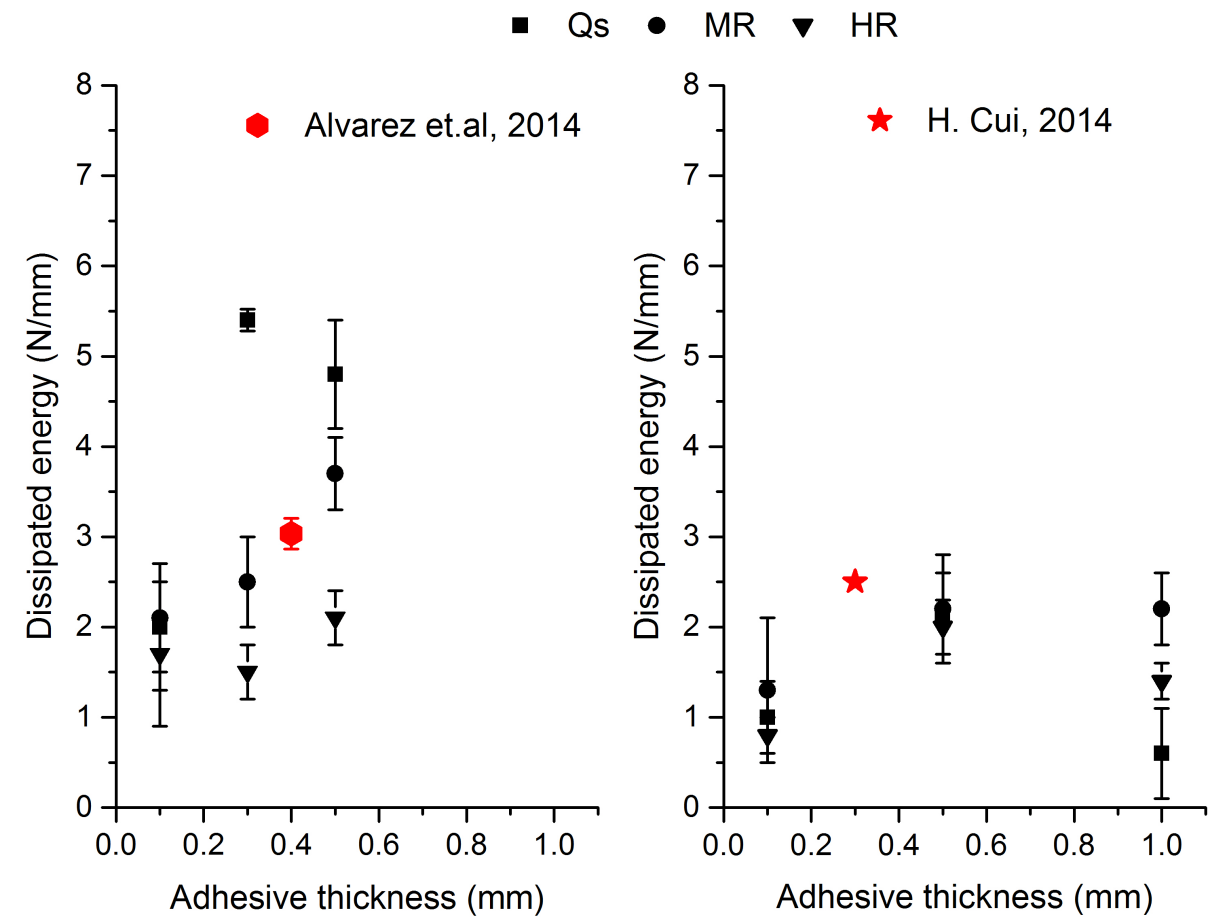

Figure 17: Dissipated energy comparison between experiments and values found in literature for: a) film adhesive AF 163-2OST and b) paste adhesive 2216 B/A Gray.

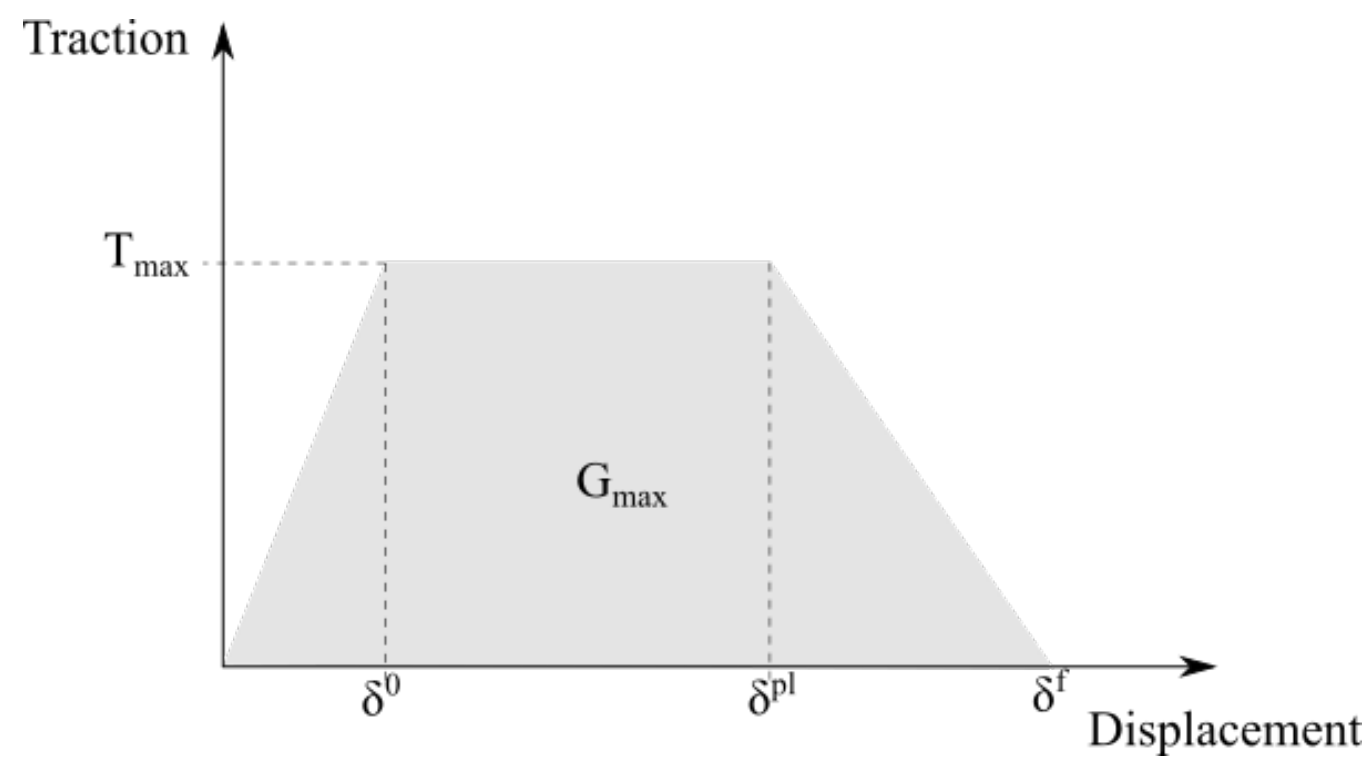

Figure 18: Trilinear traction separation law 

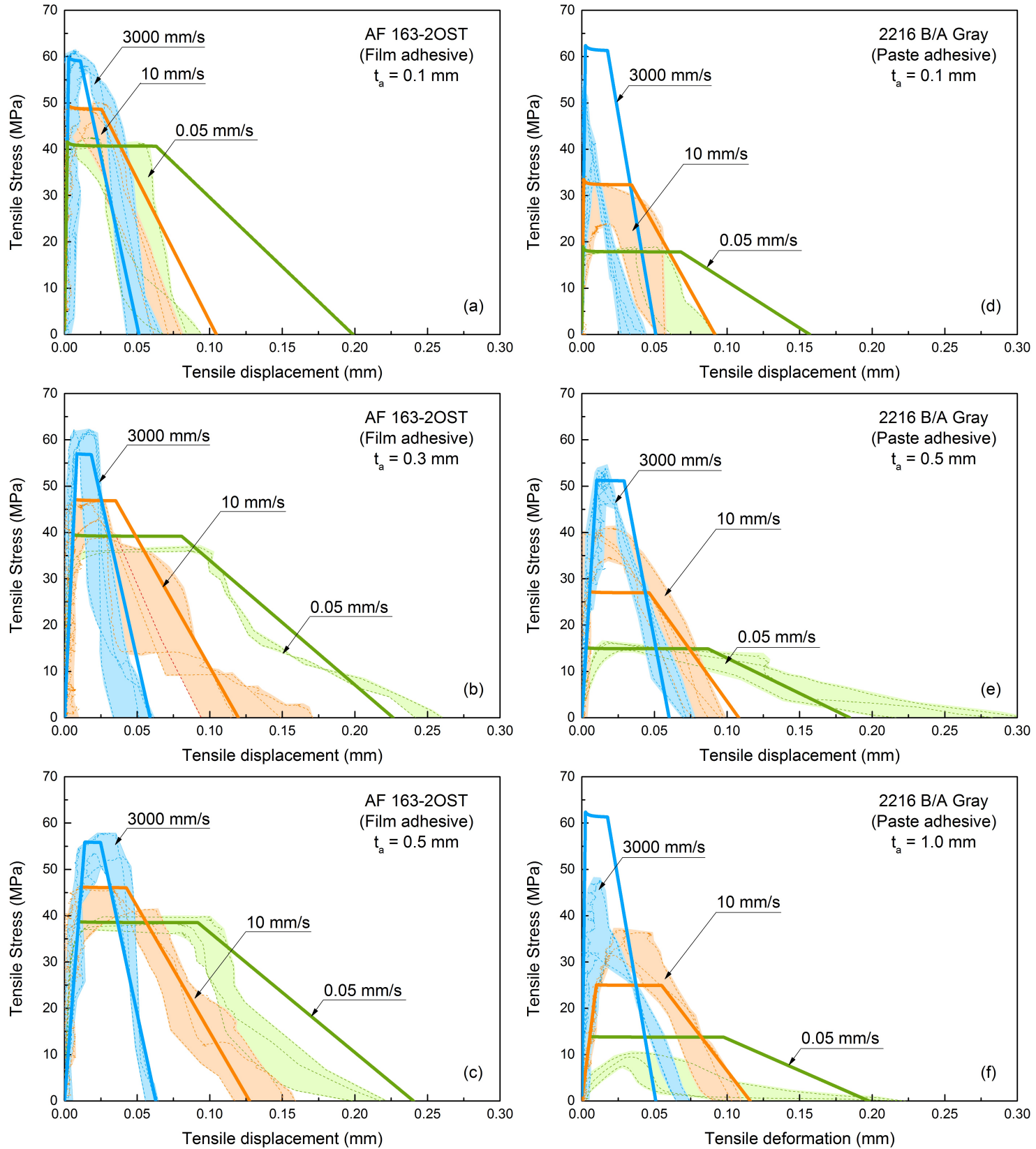

Figure 19: Results analytical model for titanium-to-titanium adhesive joints in quasi-static, medium-rate and highrate loading conditions. Predictions are made for film adhesive AF 163-2OST with (a) $t_{\mathrm{a}}=0.1 \mathrm{~mm}$, (b) $t_{\mathrm{a}}=0.3$ $\mathrm{mm}$, (c) $t_{\mathrm{a}}=0.5 \mathrm{~mm}$ and the paste adhesive $2216 \mathrm{~B} / \mathrm{A}$ Gray with (a) $t_{\mathrm{a}}=0.1 \mathrm{~mm}$, (b) $t_{\mathrm{a}}=0.5 \mathrm{~mm}$, (c) $t_{\mathrm{a}}=1.0$ $\mathrm{mm}$ 


\section{On the rate dependent behaviour of} epoxy adhesive joints: experimental characterisation and modelling of mode I failure

\section{Lißner, M.}

Elsevier

Lißner M, Alabort E, Cui $\mathrm{H}$, et al., On the rate dependent behaviour of epoxy adhesive joints: experimental characterisation and modelling of mode I failure. Composite Structures, Volume 189, 1 April 2018, Pages 286-303

http://dx.doi.org/10.1016/j.compstruct.2018.01.019

Downloaded from Cranfield Library Services E-Repository 